\title{
From Branching to Linear Time, Coalgebraically
}

\author{
Corina Cîrstea \\ Electronics and Computer Science \\ University of Southampton \\ United Kingdom \\ cc2@ecs.soton.ac.uk
}

\begin{abstract}
We consider state-based systems modelled as coalgebras whose type incorporates branching, and show that suitably adapting the definition of coalgebraic bisimulation yields a general and uniform account of the linear-time behaviour of a state in such a coalgebra. By moving away from a boolean universe of truth values, our approach can measure the extent to which a state in a system with branching is able to exhibit a particular linear-time behaviour. This instantiates to measuring the probability of a specific behaviour occurring in a probabilistic system, or measuring the minimal cost of exhibiting a given behaviour in the case of weighted computations.
\end{abstract}

Keywords: coalgebra, linear time, trace

\section{Introduction}

When analysing process behaviour, one of the early choices one has to make is between a linear and a branching view of time. In branching-time semantics, the choices a process has for proceeding from a particular state are taken into account when defining various notions of process equivalence (with bisimulation being the typical such equivalence), whereas in linear-time semantics such choices are abstracted away and the emphasis is on the individual executions that a process can exhibit. From a system verification perspective, one often chooses the linear-time view, as this not only leads to simpler specification logics and associated verification techniques, but also meets the practical need to verify all possible system executions.

Beginning with the 1990s, coalgebras have become increasingly recognised as a suitable universal model of computational systems, and more generally of dynamical systems [24]. From the outset, the 

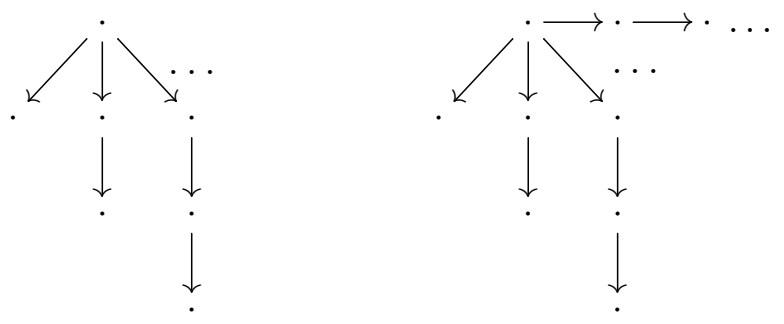

Figure 1. Two transition systems with different linear-time behaviours

theory of coalgebras was able to provide a uniform account of various bisimulation-like observational equivalences between states of a computational system (and later, also of various simulation-like behavioural preorders). However, it has so far not been equally successful in giving a generic account of the linear-time behaviour of states in systems whose type incorporates some form of branching. A generic theory of finite traces for coalgebraic models was developed by Hasuo, Jacobs and Sokolova [12]. This applies to coalgebras of type $T \circ F$, with the monad $T$ : Set $\rightarrow$ Set specifying a branching type (e.g. non-deterministic or probabilistic), and the endofunctor $F$ : Set $\rightarrow$ Set defining the structure of individual transitions (e.g. labelled transitions or successful termination). The approach in loc. cit. is complemented by that of Jacobs, Silva and Sokolova [16], where traces are derived using a determinisation procedure similar to the one for non-deterministic automata. The latter approach applies to systems modelled as coalgebras of type $G \circ \mathrm{T}$, where again a monad $\mathrm{T}$ : Set $\rightarrow$ Set is used to model branching behaviour, and an endofunctor $G$ specifies the transition structure. Neither of these approaches is, however, able to account for potentially infinite traces, which arguably provide a better semantics for linear-time behaviour - in the same way as the notion of bisimulation involves exploring the often infinite behaviour of states. A coalgebraic account of infinite traces for non-deterministic systems, modelled as coalgebras of $\mathcal{P} \circ F$ with $\mathcal{P}$ : Set $\rightarrow$ Set the powerset monad and $F$ a polynomial endofunctor, was given by Jacobs [14]. Also, a coalgebraic approach to defining infinite (as well as finite) traces for probabilistic systems, modelled as coalgebras of type $\mathcal{G} \circ F$ with $\mathcal{G}:$ Meas $\rightarrow$ Meas the Giry monad on the category of measurable spaces, was given by Kerstan and König [18]. Finally, a more general approach to defining infinite traces, which applies to both non-deterministic and probabilistic systems, also exists [1]; however, this approach fails to distinguish the two transition systems in Figure 1 (viewed as coalgebras of type $\mathcal{P} \circ(1+$ Id $)$, with 1 a one-element set and Id : Set $\rightarrow$ Set the identity functor), one with only finite maximal traces and another which can also exhibit an infinite trace. The root of the problem is the lack of a certain limit-preservation property for the powerset monad, see [1] for details. (Interestingly, the Giry monad used in the modelling of probabilistic systems is better behaved in this respect, and this results in probabilistic traces being obtained through a universal property in the Kleisli category of this monad. Both [1] and [18] follow this approach.) We also note that none of the above-mentioned approaches exploits the compositionality that is intrinsic to the coalgebraic approach. In particular, coalgebras of type $G \circ \mathrm{T} \circ F$ (of which systems with both inputs and outputs are an instance, see Example 5.14) can not be accounted for by any of the existing approaches.

This paper addresses the limitations identified above by providing a uniform and compositional treatment of (possibly infinite) linear-time behaviour in systems with branching. Our approach also provides an adequate treatment of the two systems in Figure 1. 
In the case of $T \circ F$-coalgebras over Set, working in the Kleisli category of the monad $T$ proved to provide an elegant definition of finite traces via a finality argument [12]. A similar argument, this time carried out in the Eilenberg-Moore category of the monad T, was possible for $G \circ \mathrm{T}$-coalgebras over Set [16]. However, for maximal (and hence possibly infinite) traces, a general approach based on finality appears not to exist (although, as noted earlier, this is possible for "well-behaved" monads such as the Giry monad). Here, we tackle the problem of getting a handle on the linear-time behaviour of a state in a coalgebra with branching from a different angle: we do not attempt to directly define a notion of trace equivalence between two states (e.g. via finality in some category), but focus on measuring the extent to which a state is able to exhibit a particular maximal trace. This "measuring" relates to the type of branching present in the system, and instantiates to familiar concepts such as the probability of exhibiting a given trace in probabilistic systems, the minimal cost of exhibiting a trace in weighted systems, and simply the ability to exhibit a trace in non-deterministic systems.

The technical tool for achieving this goal is a generalisation of the notions of relation and relation lifting [13], which lie at the heart of the definition of coalgebraic bisimulation. Specifically, we employ relations valued in a partial semiring, and a corresponding generalised version of relation lifting. Our approach applies to coalgebras whose type is obtained as the composition of several endofunctors on Set: one of these is a monad $\mathrm{T}$ that accounts for the presence of branching in the system, while the remaining endofunctors, assumed here to be polynomial, jointly determine the notion of linear-time behaviour. This strictly subsumes the types of systems considered in earlier work on maximal traces $[14,1,18]$, while also providing compositionality in the system type.

Our main contribution, presented in Section 5, is a uniform and compositional account of linear-time behaviour in state-based systems with branching. A by-product of our work is an extension of the study of additive monads carried out by Coumans and Jacobs [6], which itself follows earlier work by Kock [19], to what we call partially additive monads (Section 3). This generalisation of the notion of additive monad is key to our approach, as it allows systems with probabilistic branching to also be accounted for.

Our approach can be summarised as follows:

- We move from two-valued to multi-valued relations, with the universe of truth values being induced by the choice of monad for modelling branching. This instantiates to relations valued in the interval $[0,1]$ in the case of probabilistic branching, the set $\mathbb{N}^{\infty}=\mathbb{N} \cup\{\infty\}$ in the case of weighted branching, and simply $\{\perp, \top\}$ in the case of non-deterministic branching. This reflects our view that the notion of truth used to reason about the observable behaviour of a system should be dependent on the branching behaviour present in that system. Such a dependency also yields more natural temporal logics that have a conceptually simpler semantics [4]. In deriving a suitable structure on the universe of truth values, we generalise results on additive monads $[19,6]$ to partially additive monads. Specifically, we show that for a commutative, partially additive monad $\mathrm{T}$ on Set, the set T1 carries a partial semiring structure with an induced preorder, which in turn makes T1 an appropriate choice of universe of truth values.

- We generalise and adapt the notion of relation lifting used in the definition of coalgebraic bisimulation, in order to (i) support multi-valued relations, and (ii) abstract away branching. Specifically, we make use of the partial semiring structure carried by the universe of truth values to generalise relation lifting of polynomial endofunctors to multi-valued relations, and employ a canonical extension lifting induced by the monad $\mathrm{T}$ to capture a move from branching to linear time. The use of 
this extension lifting allows us to make formal the idea of measuring to what extent a state in a coalgebra with branching can exhibit a particular linear-time behaviour. Our approach resembles the idea employed by partition refinement algorithms for computing bisimulation on labelled transition systems [17]. There, one starts from a single partition of the state space, with all states related to each other, and repeatedly refines it through step-wise unfolding of the transition structure, until a fixpoint is reached. Similarly, we start by assuming that a state in a system with branching can exhibit any linear-time behaviour, and moreover, assign the maximum possible value to each pair consisting of a state and a linear-time behaviour. We then repeatedly refine the values associated to such pairs, through step-wise unfolding of the coalgebraic structure.

While our interest in this paper is in linear-time behaviour, and therefore in maximal traces, the same ideas can equally be applied to define finite trace behaviour (see Remark 5.9), thereby providing an alternative treatment of finite traces. The usefulness of such a definition and its relationship to existing accounts of finite traces $[12,16]$ is left as future work. At the same time, replacing the coalgebra of all maximal traces with a suitably-crafted coalgebra aimed at capturing error behaviour, our approach also has potential applications in verification (see Remark 5.9 for an example).

The present paper is an extended version of the conference paper [3]. It is also closely related to our previous work on maximal traces and path-based logics [2], where a game-theoretic approach to testing if a system with non-deterministic branching is able to exhibit a particular trace was described. Here we consider arbitrary branching types, and our use of fixpoints has a similar flavour to that of the graph games used in [2]. At the same time, the present work constitutes the starting point for a systematic study of coalgebraic linear-time logics, with initial results appearing in [4].

Acknowledgements Several fruitful discussions with participants at the 2012 Dagstuhl Seminar on Coalgebraic Logics helped refine the ideas presented here. Our use of relation lifting was inspired by the recent work of Hasuo on coinductive predicates [11], itself based on the seminal work of Hermida and Jacobs on the use of predicate and relation lifting in the formalisation of induction and coinduction principles [13]. Finally, the comments received from the anonymous reviewers considerably improved the presentation of the paper.

\section{Preliminaries}

\subsection{Relation Lifting}

The concepts of predicate lifting and relation lifting, to our knowledge first introduced by Hermida and Jacobs [13], are by now standard tools in the study of coalgebraic models, used e.g. to provide an alternative definition of the notion of bisimulation [15], or to describe the semantics of coalgebraic modal logics $[23,22]$. While these concepts are very general, their use so far usually restricts this generality by viewing both predicates and relations as sub-objects in some category (possibly carrying additional structure). In this paper, we make use of their full generality and move from the standard view of relations as subsets to a setting where relations are valuations into a universe of truth values. This section recalls the definition of relation lifting in the standard setting where relations are given by monomorphic spans.

Throughout this section (only), Rel denotes the category whose objects are binary relations $\left(R,\left\langle r_{1}, r_{2}\right\rangle\right)$ with $\left\langle r_{1}, r_{2}\right\rangle: R \rightarrow X \times Y$ a monomorphic span, and whose arrows from $\left(R,\left\langle r_{1}, r_{2}\right\rangle\right)$ to $\left(R^{\prime},\left\langle r_{1}^{\prime}, r_{2}^{\prime}\right\rangle\right)$ 
are given by pairs of functions $\left(f: X \rightarrow X^{\prime}, g: Y \rightarrow Y^{\prime}\right)$ s.t. $(f \times g) \circ\left\langle r_{1}, r_{2}\right\rangle$ factors through $\left\langle r_{1}^{\prime}, r_{2}^{\prime}\right\rangle$ :

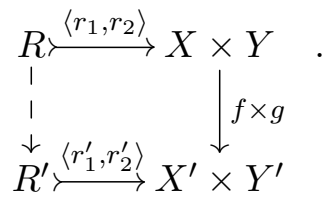

In this setting, the relation lifting of a functor $F:$ Set $\rightarrow$ Set is defined as a functor $\operatorname{Rel}(F): \operatorname{Rel} \rightarrow \operatorname{Rel}$ taking a relation $\left\langle r_{1}, r_{2}\right\rangle: R \rightarrow X \times Y$ to the relation defined by the span $\left\langle F r_{1}, F r_{2}\right\rangle: F R \rightarrow F X \times F Y$, obtained via the unique epi-mono factorisation of $\left\langle F r_{1}, F r_{2}\right\rangle$ :

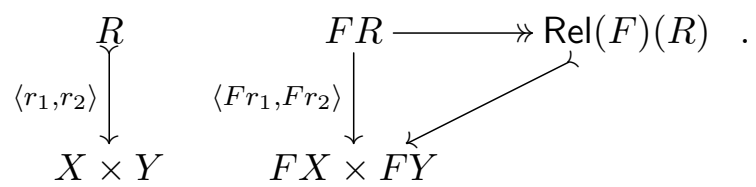

For example, if $F=\mathcal{P}$, $\operatorname{Rel}(F)$ takes a relation $R \subseteq X \times Y$ to the relation $R^{\prime} \subseteq \mathcal{P} X \times \mathcal{P} Y$ given by

$(U, V) \in R^{\prime}$ if and only if for all $x \in U$, there exists $y \in V$ such that $(x, y) \in R$, and conversely.

It follows easily that this construction is functorial, and in particular preserves the order $\leq$ between relations on the same objects given by $\left(R,\left\langle r_{1}, r_{2}\right\rangle\right) \leq\left(S,\left\langle s_{1}, s_{2}\right\rangle\right)$ if and only if $\left\langle r_{1}, r_{2}\right\rangle$ factors through $\left\langle s_{1}, s_{2}\right\rangle$ :

$$
R \underset{\left\langle r_{1}, r_{2}\right\rangle}{\stackrel{\left\langle s_{1}, s_{2}\right\rangle}{\longrightarrow}} X \times Y
$$

An alternative definition of $\operatorname{Rel}(F)$ for $F$ a polynomial functor (i.e. constructed from the identity and constant functors using finite products and set-indexed coproducts) can be given by induction on the structure of $F$. We refer the reader to [15, Section 3.1] for details of this definition. An extension of this definition to a more general notion of relation will be given in Section 4.

\subsection{Coalgebras}

We model state-based, dynamical systems as coalgebras over the category of sets. Given a functor $F: \mathbb{C} \rightarrow \mathbb{C}$ on an arbitrary category, an $F$-coalgebra is given by a pair $(C, \gamma)$ with $C$ an object of $\mathbb{C}$, used to model the state space, and $\gamma: C \rightarrow F C$ a morphism in $\mathbb{C}$, describing the one-step evolution of the system states. Then, a canonical notion of observational equivalence between the states of two $F$-coalgebras is provided by the notion of bisimulation. Of the many, and under the assumption that $F$ preserves weak pullbacks, equivalent definitions of bisimulation (see [15] for a detailed account), we recall the one based on relation lifting. This applies to coalgebras over the category of sets (as described below), but also more generally to categories with so-called logical factorisation systems ${ }^{1}$ (as described

${ }^{1}$ These are factorisation systems $(\mathcal{M}, \mathcal{E})$ with the additional assumptions that (i) the maps in $\mathcal{M}$ are monos, (ii) $\mathcal{M}$ is closed under pullback, and (iii) pullbacks of maps in $\mathcal{E}$ along maps in $\mathcal{M}$ are themselves in $\mathcal{E}$. 
in [15]). According to this definition, an F-bisimulation between coalgebras $(C, \gamma)$ and $(D, \delta)$ over Set is a $\operatorname{Rel}(F)$-coalgebra $(R, \rho)$ :

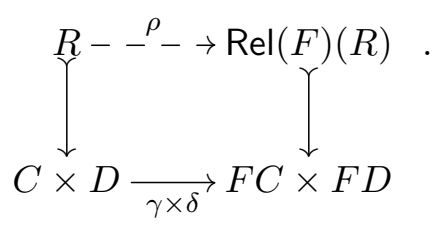

In the remainder of this section we outline a coalgebraic generalisation of a well-known partition refinement algorithm for computing bisimilarity (i.e. the largest bisimulation) on finite-state labelled transition systems [17] (see also [25]). For an arbitrary endofunctor $F$ : Set $\rightarrow$ Set and two finite-state $F$ coalgebras $(C, \gamma)$ and $(D, \delta)$, the generalised algorithm iteratively computes increasingly finer relations $\simeq_{i} \subseteq C \times D$ with $i=0,1, \ldots$ as follows:

- $\simeq_{0}=C \times D$

- $\simeq_{i+1}=(\gamma \times \delta)^{*}\left(\operatorname{Rel}(F)\left(\simeq_{i}\right)\right)$ for $i=0,1, \ldots$

where $(\gamma \times \delta)^{*}$ takes a relation $R \subseteq F C \times F D$ to the relation $R^{\prime}=\{(c, d) \in C \times D \mid(\gamma(c), \delta(d)) \in R\}$; that is, $R^{\prime}$ is obtained by taking the pullback of $R \succ F C \times F D$ along $C \times D \stackrel{\gamma \times \delta}{\longrightarrow} F C \times F D$ :

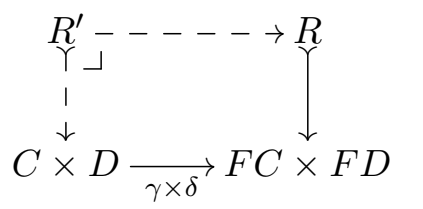

Thus, in the initial approximation $\simeq_{0}$ of the bisimilarity relation, all states are related, whereas at step $i+1$ two states are related if and only if their one-step observations are related using the relation $\operatorname{Rel}(F)\left(\simeq_{i}\right)$. Given the finite state space assumption, the chain of relations $\simeq_{0} \supseteq_{1} \supseteq$.. stabilises after a finite number of steps. Bisimilarity between the coalgebras $(C, \gamma)$ and $(D, \delta)$ thus arises as the greatest fixpoint of a monotone operator on the complete lattice of relations between $C$ and $D$, which takes a relation $R \subseteq C \times D$ to the relation $(\gamma \times \delta)^{*}(\operatorname{Rel}(F)(R))$. This characterisation extends to coalgebras with infinite state spaces, however, in this case the fixpoint can not, in general, be reached in a finite number of steps (see [25, Section 2] for the case of labelled transition systems with infinite state spaces).

The above greatest fixpoint characterisation of bisimilarity will be adapted in Section 5, in order to characterise the extent to which a state in a coalgebra with branching can exhibit a given linear-time behaviour. Therein, the two coalgebras in question will have different types: one of the coalgebras will incorporate branching behaviour and will be used to model the system of interest, whereas the other will have linear behaviour only and will be used to describe the domain of possible traces. 


\subsection{Monads}

In what follows, we use monads $(\mathrm{T}, \eta, \mu)$ on Set to capture branching in coalgebraic types. Here, $\eta$ : $\mathrm{Id} \Rightarrow \mathrm{T}$ and $\mu: \mathrm{T} \circ \mathrm{T} \Rightarrow \mathrm{T}$ are the unit and multiplication of $\mathrm{T}$, subject to the following laws:
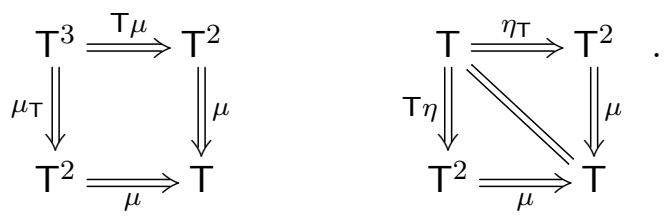

Moreover, we assume that these monads are strong and commutative. A strong monad comes equipped with a strength map st $X, Y: X \times \mathrm{T} Y \rightarrow \mathrm{T}(X \times Y)$, natural in $X$ and $Y$ and subject to coherence conditions w.r.t. $\eta$ and $\mu$ (see e.g. [15, Section 5.2]). For such a monad, one can also define a swapped strength map $\mathrm{st}_{X, Y}^{\prime}: \mathrm{T} X \times Y \rightarrow \mathrm{T}(X \times Y)$ by:

$$
\mathrm{T} X \times Y \stackrel{\mathrm{tw}_{\mathrm{T} X, Y}}{\longrightarrow} Y \times \mathrm{T} X \stackrel{\mathrm{st}_{Y, X}}{\longrightarrow} \mathrm{T}(Y \times X) \stackrel{\mathrm{Ttw}_{Y, X}}{\longrightarrow} \mathrm{T}(X \times Y)
$$

where $\operatorname{tw}_{X, Y}: X \times Y \rightarrow Y \times X$ is the twist map taking $(x, y) \in X \times Y$ to $(y, x)$. Commutative monads are strong monads where the maps $\mu_{X \times Y} \circ \mathrm{Tst}_{X, Y}^{\prime} \circ$ st $_{\mathrm{T} X, Y}: \mathrm{T} X \times \mathrm{T} Y \rightarrow \mathrm{T}(X \times Y)$ and $\mu_{X \times Y} \circ \mathrm{Tst}_{X, Y} \circ \mathrm{st}_{X, \mathrm{~T} Y}^{\prime}: \mathrm{T} X \times \mathrm{T} Y \rightarrow \mathrm{T}(X \times Y)$ coincide, yielding a double strength map dst $_{X, Y}: \mathrm{T} X \times \mathrm{T} Y \rightarrow \mathrm{T}(X \times Y)$ for each choice of sets $X, Y$.

Example 2.1. As examples of (commutative) monads, we consider:

1. the powerset monad $\mathcal{P}:$ Set $\rightarrow$ Set, modelling non-deterministic computations and given by

$$
\mathcal{P} X=\{U \mid U \subseteq X\} \quad(\mathcal{P} f)(U)=\{f(x) \mid x \in U\}
$$

for $f: X \rightarrow Y$ and $U \in \mathcal{P} X$. Its unit is given by singletons whereas its multiplication is given by unions. Its strength and double strength are given by

$$
\text { st }_{X, Y}(x, V)=\{x\} \times V \quad \operatorname{dst}_{X, Y}(U, V)=U \times V
$$

for $x \in X, U \in \mathcal{P} X$ and $V \in \mathcal{P} Y$.

2. the semiring monad $\mathrm{T}_{S}$ : Set $\rightarrow$ Set, with $(S,+, 0, \bullet, 1)$ a commutative semiring, given by

$$
\mathrm{T}_{S} X=\{\varphi: X \rightarrow S \mid \sup (\varphi) \text { is finite }\} \quad\left(\mathrm{T}_{S} f\right)(\varphi)(y)=\sum_{x \in \sup (\varphi), f(x)=y} \varphi(x)
$$

for $f: X \rightarrow Y, \varphi \in \mathrm{T}_{S} X$ and $y \in Y$, where $\sup (\varphi)=\{x \in X \mid \varphi(x) \neq 0\}$ is the support of $\varphi$. Its unit and multiplication are given by

$$
\eta_{X}(x)(y)=\left\{\begin{array}{ll}
1 & \text { if } y=x \\
0 & \text { otherwise }
\end{array} \quad \mu_{X}(\Phi)=\sum_{\varphi \in \sup (\Phi)} \sum_{x \in \sup (\varphi)} \Phi(\varphi) \bullet \varphi(x)\right.
$$


for $x, y \in X$ and $\Phi \in S^{\left(S^{X}\right)}$, while its strength and double strength are given by

$$
\text { st }_{X, Y}(x, \psi)(z, y)=\left\{\begin{array}{ll}
\psi(y) & \text { if } z=x \\
0 & \text { otherwise }
\end{array} \quad \operatorname{dst}_{X, Y}(\varphi, \psi)(z, y)=\varphi(z) \bullet \psi(y)\right.
$$

for $x, z \in X, \varphi \in \mathrm{T}_{S} X, \psi \in \mathrm{T}_{S} Y$ and $y \in Y$. As a concrete example, we will consider the semiring $W=\left(\mathbb{N}^{\infty}, \min , \infty,+, 0\right)$ (sometimes referred to as the tropical semiring), and use $\mathrm{T}_{W}$ to model weighted computations. We also note that, while the finite powerset monad on Set can be recovered as a particular case of the semiring monad $\mathrm{T}_{S}$ (namely by taking $S=$ $(\{\perp, \top\}, \vee, \perp, \wedge, \top))$, the full powerset monad can not (because of the finite support restriction in the definition of $\mathrm{T}_{S}$ ).

3. the sub-probability distribution monad $\mathcal{S}:$ Set $\rightarrow$ Set, modelling probabilistic computations and given by

$$
\mathcal{S} X=\left\{\varphi: X \rightarrow[0,1] \mid \sum_{x \in \sup (\varphi)} \varphi(x) \leq 1\right\} \quad(\mathcal{S} f)(\varphi)(y)=\sum_{x \in \sup (\varphi), f(x)=y} \varphi(x)
$$

for $f: X \rightarrow Y, \varphi \in \mathcal{S} X$ and $y \in Y$. Its unit, multiplication, strength and double strength are defined similarly to those of the semiring monad, only with the semiring operations replaced by + and $*$ respectively. We note that the above definition of $\mathcal{S}$ also allows for sub-probability distributions with countable support.

4. the lift monad $\mathcal{L}:$ Set $\rightarrow$ Set, modelling exceptions, and given by

$$
\mathcal{L} X=\{\perp\}+X \quad \mathcal{L} f=\mathrm{id}_{\{\perp\}}+f
$$

for $f: X \rightarrow Y$. Its unit is given by the coproduct injections $\iota_{2}: X \rightarrow \mathcal{L} X$, whereas its multiplication takes both $\iota_{1}(\perp)$ and $\iota_{2}\left(\iota_{1}(\perp)\right)$ to $\perp$, and $\iota_{2}\left(\iota_{2}(x)\right)$ to $\iota_{2}(x)$, for $x \in X$. Its strength and double strength are given by

$$
\begin{array}{r}
\operatorname{st}_{X, Y}\left(x, \iota_{1}(\perp)\right)=\iota_{1}(\perp) \quad \operatorname{st}_{X, Y}\left(x, \iota_{2}(y)\right)=\iota_{2}(x, y) \\
\text { dst }_{X, Y}\left(\iota_{1}(\perp), \iota_{1}(\perp)\right)=\operatorname{dst}_{X, Y}\left(\iota_{1}(\perp), \iota_{2}(y)\right)=\operatorname{dst}_{X, Y}\left(\iota_{1}(x), \iota_{2}(\perp)\right)=\iota_{1}(\perp) \\
\operatorname{dst}_{X, Y}\left(\iota_{2}(x), \iota_{2}(y)\right)=\iota_{2}(x, y)
\end{array}
$$

for $x \in X$ and $y \in Y$.

\section{From Partially Additive, Commutative Monads to Partial Commuta- tive Semirings with Order}

Later in this paper we will consider coalgebras whose type is given by the composition of several endofunctors on Set, one of which is a commutative monad T : Set $\rightarrow$ Set accounting for the presence of branching in the systems of interest. This section builds on results by Kock [19], and by Coumans and Jacobs [6], in order to derive a suitably-structured universe of truth values from such a monad. The assumption of loc. cit. concerning the additivity of the monad under consideration is here weakened to 
partial additivity (see Definition 3.2); this allows us to incorporate the sub-probability distribution monad and the lift monad (neither of which is additive) into our framework. Specifically, we show that any commutative, partially additive monad $\mathrm{T}:$ Set $\rightarrow$ Set induces a partial commutative semiring structure on the set T1, with $1=\{*\}$ a final object in Set. We recall that a commutative semiring consists of a set $S$ carrying two commutative monoid structures $(+, 0)$ and $(\bullet, 1)$, with the latter distributing over the former: $s \bullet 0=0$ and $s \bullet(t+u)=s \bullet t+s \bullet u$ for all $s, t, u \in S$. A partial commutative semiring is defined similarly, except that + is now a partial operation subject to the condition that whenever $t+u$ is defined, then so is $s \bullet t+s \bullet u$, and moreover $s \bullet(t+u)=s \bullet t+s \bullet u$. The relevance of a partial commutative semiring structure on the set of truth values will become clear in Sections 4 and 5.

It follows from results in [6] that any commutative monad $(T, \eta, \mu)$ on Set induces a commutative monoid $\left(\mathrm{T} 1, \bullet, \eta_{1}(*)\right)$, with multiplication $\bullet: \mathrm{T} 1 \times \mathrm{T} 1 \rightarrow \mathrm{T} 1$ given by the composition

$$
\mathrm{T} 1 \times \mathrm{T} 1 \stackrel{\mathrm{dst}_{1,1}}{\longrightarrow} \mathrm{T}(1 \times 1) \stackrel{\mathrm{T} \pi_{2}}{\longrightarrow} \mathrm{T} 1
$$

Alternatively, this multiplication can be defined as the composition

$$
\mathrm{T} 1 \times \mathrm{T} 1 \stackrel{\mathrm{st}_{1, \mathrm{~T} 1}^{\prime}}{\longrightarrow} \mathrm{T}(1 \times \mathrm{T} 1) \stackrel{\mathrm{T} \pi_{2}}{\longrightarrow} \mathrm{T}^{2} 1 \stackrel{\mu_{1}}{\longrightarrow} \mathrm{T} 1
$$

or as

$$
\mathrm{T} 1 \times \mathrm{T} 1 \stackrel{\mathrm{st}_{\mathrm{T} 1,1}}{\longrightarrow} \mathrm{T}(\mathrm{T} 1 \times 1) \stackrel{\mathrm{T} \pi_{1}}{\longrightarrow} \mathrm{T}^{2} 1 \stackrel{\mu_{1}}{\longrightarrow} \mathrm{T} 1 .
$$

(While the previous two definitions coincide for commutative monads, this is not the case in general.)

Remark 3.1. The following maps define left and right actions of $(T 1, \bullet)$ on $T X$ :

$$
\mathrm{T} 1 \times \mathrm{T} X \stackrel{\mathrm{dst}_{1, X}}{\longrightarrow} \mathrm{T}(1 \times X) \stackrel{\mathrm{T} \pi_{2}}{\longrightarrow} \mathrm{T} X \quad \mathrm{~T} X \times \mathrm{T} 1 \stackrel{\mathrm{dst}_{X, 1}}{\longrightarrow} \mathrm{T}(X \times 1) \stackrel{\mathrm{T} \pi_{1}}{\longrightarrow} \mathrm{T} X
$$

On the other hand, any monad $\mathrm{T}:$ Set $\rightarrow$ Set with $\mathrm{T} \emptyset=1$ is such that, for any $X, \mathrm{~T} X$ has a zero element $0 \in \mathrm{T} X$, obtained as $\left(\mathrm{T} ?_{X}\right)(*)$, with the map $?_{X}: \emptyset \rightarrow X$ arising by initiality. This yields a zero map $0: Y \rightarrow \mathrm{T} X$ for any $X, Y$, obtained as the composition

$$
Y \stackrel{!_{Y}}{\longrightarrow} \mathrm{T} \emptyset \stackrel{\mathrm{T} ?_{X}}{\longrightarrow} \mathrm{T} X
$$

with the map $!_{Y}: Y \rightarrow \mathrm{T} \emptyset$ arising by finality. Now consider the unique map $\delta_{X, Y}=\left\langle\mu_{X} \circ \mathrm{T} p_{1}, \mu_{Y} \circ\right.$ $\left.\mathrm{T} p_{2}\right\rangle: \mathrm{T}(X+Y) \rightarrow \mathrm{T} X \times \mathrm{T} Y$ making the following diagram commute:

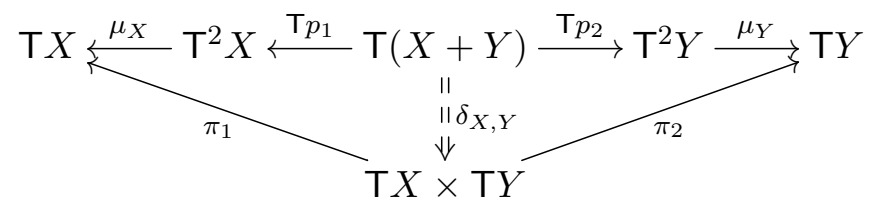

where $p_{1}=\left[\eta_{X}, 0\right]: X+Y \rightarrow \mathrm{T} X$ and $p_{2}=\left[0, \eta_{Y}\right]: X+Y \rightarrow \mathrm{T} Y$. [6, Lemma 15, (i)] shows that the maps $\delta_{X, Y}$ define a natural transformation. 


\section{Definition 3.2. (Partially additive monad)}

A monad $T$ : Set $\rightarrow$ Set is called partially additive if $T \emptyset=1$ and moreover, the natural transformation $\delta$ arising from (1) is a natural monomorphism.

Remark 3.3. If $\delta$ is a natural isomorphism, then $\mathrm{T}$ is called additive. Additive monads were studied in $[19,6]$. Additivity can be regarded as a generalisation of the isomorphism $\mathcal{P}(X+Y) \simeq \mathcal{P} X \times \mathcal{P} Y$ (natural in $X$ and $Y$ ) to an arbitrary monad on Set, whereas partial additivity captures a similar property of the sub-probability monad. The latter is clearly not additive, since two sub-probability distributions, over $X$ and respectively $Y$, can not always be amalgamated into a sub-probability distribution over $X+Y$. However, any sub-probability distribution over $X+Y$ naturally induces a pair of sub-probability distributions, over $X$ and respectively $Y$, and moreover, this map is mono. A similar observation holds for the lift monad, which, like the sub-probability distribution monad, is not additive.

Example 3.4. All the monads in Example 2.1 are partially additive.

1. For $\mathrm{T}=\mathcal{P}$, the zero map $0: Y \rightarrow \mathcal{P} X$ takes $y \in Y$ to $\emptyset \in \mathcal{P} X$, and $\delta_{X, Y}: \mathcal{P}(X+Y) \rightarrow$ $\mathcal{P} X \times \mathcal{P} Y$ takes $Z \subseteq X+Y$ to $\left(\left(\iota_{1}\right)^{-1}(Z),\left(\iota_{2}\right)^{-1}(Z)\right) \in \mathcal{P} X \times \mathcal{P} Y$.

2. For $\mathrm{T}=\mathrm{T}_{S}$ with $(S,+, 0, \bullet, 1)$ a commutative semiring, the zero map $0: Y \rightarrow \mathrm{T}_{S} X$ takes $y \in Y$ to the map $x \mapsto 0$ for $x \in X$, whereas $\delta_{X, Y}: S^{X+Y} \rightarrow S^{X} \times S^{Y}$ takes $\varphi \in S^{X+Y}$ to $\left(\varphi \circ \iota_{1}, \varphi \circ \iota_{2}\right) \in S^{X} \times S^{Y}$.

3. For $\mathrm{T}=\mathcal{S}$, the zero map $0: Y \rightarrow \mathcal{S} X$ takes $y \in Y$ to the sub-probability distribution $x \mapsto 0$ for $x \in X$, whereas $\delta_{X, Y}: \mathcal{S}(X+Y) \rightarrow \mathcal{S} X \times \mathcal{S} Y$ takes $\varphi \in \mathcal{S}(X+Y)$ to $\left(\varphi \circ \iota_{1}, \varphi \circ \iota_{2}\right) \in \mathcal{S} X \times \mathcal{S} Y$. (Since $\varphi$ is a sub-probability distribution, so are $\varphi \circ \iota_{1}$ and $\varphi \circ \iota_{2}$.)

4. For $\mathrm{T}=\mathcal{L}$, the zero map $0: Y \rightarrow \mathcal{L} X$ takes $y \in Y$ to $\iota_{1}(\perp)$, whereas $\delta_{X, Y}:\{\perp\}+(X+Y) \rightarrow$ $(\{\perp\}+X) \times(\{\perp\}+Y)$ takes $\iota_{1}(\perp)$ to $\left(\iota_{1}(\perp), \iota_{1}(\perp)\right), \iota_{2}\left(\iota_{1}(x)\right)$ to $\left(\iota_{2}(x), \iota_{1}(\perp)\right)$, and $\iota_{2}\left(\iota_{2}(y)\right)$ to $\left(\iota_{1}(\perp), \iota_{2}(y)\right)$.

In the first two cases, $\delta_{X, Y}$ is an isomorphism, whereas in the last two cases, for $X \neq \emptyset$ and $Y \neq \emptyset, \delta_{X, Y}$ is only a monomorphism.

For a (partially) additive monad $\mathrm{T}$, the (partial) inverse $q_{X, X}: \mathrm{T} X \times \mathrm{T} X \rightarrow \mathrm{T}(X+X)$ of the map $\delta_{X, X}: \mathrm{T}(X+X) \rightarrow \mathrm{T} X \times \mathrm{T} X$ can be used to define a (partial) addition on the set $\mathrm{T} X$, given by $\mathrm{T}\left[1_{X}, 1_{X}\right] \circ q_{X, X}:$

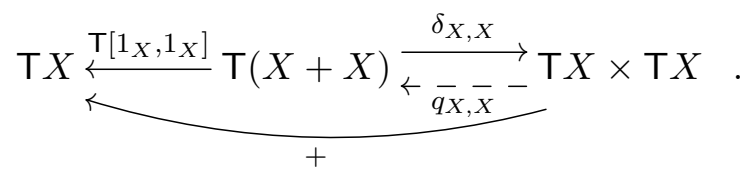

That is, $a+b$ is defined if and only if $(a, b) \in \operatorname{Im}\left(\delta_{X, X}\right)$. A similar, but total, addition operation is defined in $[19,6]$ for additive monads.

Coumans and Jacobs [6, Section 5.2] explore the connection between additive, commutative monads and commutative semirings. The next result provides a generalisation to partially additive, commutative monads and partial commutative semirings. Its proof is an adaptation of the corresponding proofs in [6].

Proposition 3.5. Let $\mathrm{T}$ be a commutative, (partially) additive monad. Then: 
1. $\left(\mathrm{T} 1, \bullet, \eta_{1}(*)\right)$ is a commutative monoid.

2. $(\mathrm{T} X, 0,+)$ is a (partial) commutative monoid, for each set $X$.

3. $\left(\mathrm{T} 1,0,+, \bullet, \eta_{1}(*)\right)$ is a (partial) commutative semiring.

\section{Proof:}

Statement 1 follows directly from [6, Lemma 10], using the commutativity of T.

The proof of 2 is similar to that of [6, Lemma 17, (i) and (ii)]. To show that 0 is a right unit for + , consider the following diagram:

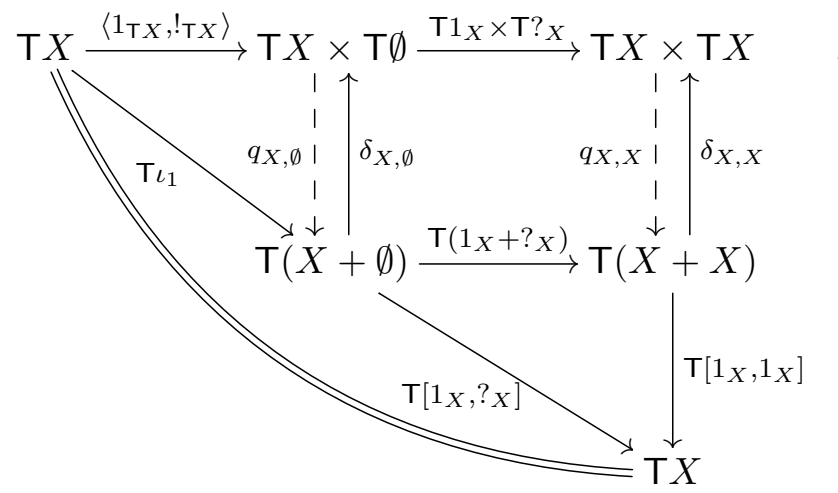

The top-right rectangle of this diagram (solid lines) commutes as a result of the (bi)naturality of $\delta$, proved in [6, Lemma 15, (i)] for any monad $\mathrm{T}$ such that $\mathrm{T} \emptyset=1$; concretely, $(\mathrm{T} f \times \mathrm{T} g) \circ \delta_{X, Y}=\delta_{U, V} \circ \mathrm{T}(f+g)$ for any $f: U \rightarrow X$ and $g: V \rightarrow Y$. Commutativity of the rest of the diagram (solid lines) follows directly from the definition of $\delta_{X, \emptyset}$ (top-left triangle) and from standard properties of coproducts. Finally, since $\delta_{X, \emptyset}$ is an isomorphism (as $\left.\mathrm{T} \emptyset=1\right)$, so is $q_{X, \emptyset}$, and therefore $x+0=\left(\mathrm{T}\left[1_{X}, 1_{X}\right] \circ q_{X, X} \circ\left(\mathrm{T} 1_{X} \times\right.\right.$ $\left.\left.\mathrm{T} ?_{X}\right) \circ\left\langle 1_{\mathrm{T} X}, !_{\mathrm{T} X}\right\rangle\right)(x)$ is always defined, and equal to $x \in \mathrm{T} X$.

The commutativity of + (whenever one of the sides of the commutativity law is defined) follows from the commutativity of the following diagram (solid lines):



where the commutativity of the left rectangle (solid lines) follows from [6, Lemma 15, (ii)] (which only requires $\mathrm{T} \emptyset=1$ ). This, in turn, results in $b+a$ (given by $\left(\mathrm{T}\left[1_{X}, 1_{X}\right] \circ q_{X, X} \circ\left\langle\pi_{2}, \pi_{1}\right\rangle\right)(a, b)$ ) being defined whenever $a+b$ (given by $\left.\left(\mathrm{T}\left[1_{X}, 1_{X}\right] \circ q_{X, X}\right)(a, b)\right)$ is defined, which then yields commutativity of the left rectangle (dashed lines) and therefore commutativity of the outer rectangle. The latter finally gives $a+b=b+a$ whenever $a+b$ is defined.

The associativity of + (whenever one of the sides of the associativity law is defined) follows similarly, although the proof is somewhat more involved. 
Finally, the proof of 3 relies on the commutativity of the following diagram (solid lines):



where $a_{\mathrm{T} X}: \mathrm{T} X \times \mathrm{T} 1 \rightarrow \mathrm{T} X$ is the right action from Remark 3.1. The composition $\bullet \circ\left(\mathrm{T}\left[1_{X}, 1_{X}\right] \times\right.$ $\left.1_{\mathrm{T} 1}\right) \circ\left(q_{1,1} \times 1_{\mathrm{T} 1}\right)$ captures the computation of $(a+b) \bullet c$, whereas the composition $\mathrm{T}\left[1_{X}, 1_{X}\right] \circ q_{1,1} \circ(\bullet \times$ $\bullet) \circ\left\langle\pi_{1} \times \mathrm{id}, \pi_{2} \times \mathrm{id}\right\rangle$ captures the computation $a \bullet c+b \bullet c$, with $a, b, c \in \mathrm{T} 1$. The fact that $\delta$ commutes with the strength map (by [6, Lemma 15, (iv)]), together with $a_{\mathrm{T}(1+1)}$ and $\bullet$ being essentially given by the double strength maps dst ${ }_{1+1,1}$ and $\mathrm{dst}_{1,1}$, yields $(\bullet \times \bullet) \circ\left\langle\pi_{1} \times \mathrm{id}, \pi_{2} \times \mathrm{id}\right\rangle \circ\left(\delta \times 1_{\mathrm{T} 1}\right)=\delta \circ a_{\mathrm{T}(1+1)}$, that is, commutativity (via the solid lines) of the right side of the above diagram. This immediately results in $a \bullet c+b \bullet c$ being defined whenever $a+b$ is defined, and hence in the commutativity of the right side of the diagram also via the dashed lines. This, combined with the commutativity of the left side of the diagram (which is simply the naturality of the right action $a$ ), gives $(a+b) \bullet c=a \bullet c+b \bullet c$ whenever $a+b$ is defined.

Example 3.6. For the monads in Example 2.1, one obtains the commutative semirings $(\{\perp, \top\}, \vee, \perp, \wedge, \top)$ when $\mathrm{T}=\mathcal{P}$ and $W=\left(\mathbb{N}^{\infty}\right.$, min, $\left.\infty,+, 0\right)$ when $\mathrm{T}=\mathrm{T}_{W}$, and the partial commutative semirings $([0,1],+, 0, *, 1)$ when $\mathrm{T}=\mathcal{S}$ (with $a+b$ defined if and only if $a+b \leq 1)$ and $(\{0,1\},+, 0, *, 1)$ when $\mathrm{T}=\mathcal{L}$ (where for convenience we now write 0 for $\iota_{1}(\perp)$ ), and where again, $a+b$ is defined if and only if $a+b \leq 1$ ). Moreover, it is a straightforward calculation to show that, for any commutative semiring $(S,+, 0, \bullet, 1)$, the semiring induced by the monad $\mathrm{T}_{S}$ coincides with $(S,+, 0, \bullet, 1)$.

\section{Generalised Relations and Relation Lifting}

This section introduces generalised relations valued in a partial commutative semiring, and shows how to lift polynomial endofunctors on Set to the category of generalised relations. We begin by fixing a partial commutative semiring $(S,+, 0, \bullet, 1)$, and noting that the partial monoid $(S,+, 0)$ can be used to define a preorder relation on $S$ as follows:

$$
x \sqsubseteq y \quad \text { if and only if there exists } z \in S \text { such that } x+z=y
$$

for $x, y \in S$. It is then straightforward to show (using the definition of a partial commutative semiring) that the preorder $\sqsubseteq$ has $0 \in S$ as bottom element, and is preserved by $\bullet$ in each argument. Proper (i.e. not partial) semirings where the preorder $\sqsubseteq$ is a partial order are called naturally ordered [8].

Example 4.1. For the monads in Example 2.1, the preorders associated to the induced (partial) semirings (see Example 3.6) are all partial orders: $\leq$ on $\{\perp, \top\}$ for $T=\mathcal{P}, \geq$ on $\mathbb{N}^{\infty}$ for $T=T_{W}, \leq$ on $[0,1]$ for $\mathrm{T}=\mathcal{S}$, and $\leq$ on $\{0,1\}$ for $\mathrm{T}=\mathcal{L}$. 
We now let Rel denote the category ${ }^{2}$ with objects given by triples $(X, Y, R)$, where $R: X \times Y \rightarrow S$ is a function defining a multi-valued relation (or $S$-valued relation), and with arrows from $(X, Y, R)$ to $\left(X^{\prime}, Y^{\prime}, R^{\prime}\right)$ given by pairs of functions $(f, g)$ as below, such that $R \sqsubseteq R^{\prime} \circ(f \times g)$ :

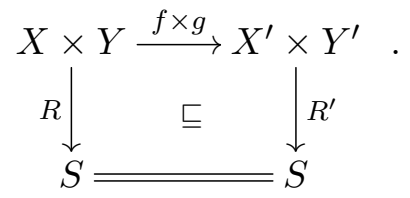

Here, the order $\sqsubseteq$ on $S$ has been extended pointwise to $S$-valued relations with the same carrier.

We write $\operatorname{Rel}_{X, Y}$ for the fibre over $(X, Y)$, i.e. the subcategory of Rel with objects given by $S$ valued relations over $X \times Y$ and arrows given by $\left(1_{X}, 1_{Y}\right)$. It is straightforward to check that the functor $q:$ Rel $\rightarrow$ Set $\times$ Set taking $(X, Y, R)$ to $(X, Y)$ defines a fibration [15]: the reindexing functor $(f, g)^{*}: \operatorname{Rel}_{X^{\prime}, Y^{\prime}} \rightarrow \operatorname{Rel}_{X, Y}$ takes $R^{\prime}: X^{\prime} \times Y^{\prime} \rightarrow S$ to $R^{\prime} \circ(f \times g): X \times Y \rightarrow S$.

We now proceed to generalising relation lifting to $S$-valued relations.

\section{Definition 4.2. (Generalised relation lifting)}

Let $F:$ Set $\rightarrow$ Set. A relation lifting of $F$ is a functor ${ }^{3} \Gamma: \operatorname{Rel} \rightarrow \operatorname{Rel}$ such that $q \circ \Gamma=(F \times F) \circ q$ :

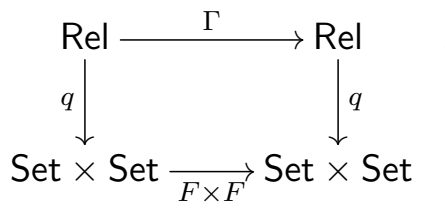

We immediately note a fundamental difference compared to standard relation lifting as defined in Section 2.1. While in the case of standard relations each functor admits exactly one lifting, Definition 4.2 implies neither the existence nor the uniqueness of a lifting. We defer the study of a canonical lifting (similar to $\operatorname{Rel}(F)$ in the case of standard relations) to future work, and show how to define a relation lifting of $F$ in the case when $F$ is a polynomial functor. To this end, we make the additional assumption that the unit 1 of the semiring multiplication is a top element (which we also write as $T$ ) for the preorder $\sqsubseteq$. Recall that $\sqsubseteq$ also has a bottom element (which we will sometimes denote by $\perp$ ), given by the unit 0 of the (partial) semiring addition. The definition of the relation lifting of a polynomial functor $F$ is by structural induction on $F$ and makes use of the partial semiring structure on $S$ :

- If $F=$ Id, $\operatorname{Rel}(F)$ takes an $S$-valued relation to itself.

- If $F=C, \operatorname{Rel}(F)$ takes an $S$-valued relation to the equality relation $\mathrm{Eq}(C): C \times C \rightarrow S$ given by

$$
\mathrm{Eq}_{C}\left(c, c^{\prime}\right)=\left\{\begin{array}{l}
\top \text { if } c=c^{\prime} \\
\perp \text { otherwise }
\end{array}\right.
$$

\footnotetext{
${ }^{2}$ To keep notation simple, the dependency on $S$ is left implicit.

${ }^{3}$ Given the definition of the fibration $q$, such a functor is automatically a morphism of fibrations.
} 
- If $F=F_{1} \times F_{2}, \operatorname{Rel}(F)$ takes an $S$-valued relation $R: X \times Y \rightarrow S$ to:

$$
\left(F_{1} X \times F_{2} X\right) \times\left(F_{1} Y \times F_{2} Y\right) \stackrel{\left\langle\pi_{1} \times \pi_{1}, \pi_{2} \times \pi_{2}\right.}{\longrightarrow}\left(F_{1} X \times F_{1} Y\right) \times\left(F_{2} X \times F_{2} Y\right) \stackrel{\operatorname{Rel}\left(F_{1}\right)(R) \times \operatorname{Rel}\left(F_{2}\right)(R)}{\longrightarrow} S \times S \stackrel{\bullet}{\longrightarrow} S
$$

The functoriality of this definition follows from the preservation of $\sqsubseteq$ by $\bullet$ (see Section 3 ).

- if $F=\coprod_{i \in I} F_{i}, \operatorname{Rel}(F)(R):\left(\coprod_{i \in I} F_{i} X\right) \times\left(\coprod_{i \in I} F_{i} Y\right) \rightarrow S$ is defined by case analysis:

$$
\operatorname{Rel}(F)(R)\left(\iota_{i}(u), \iota_{j}(v)\right)= \begin{cases}\operatorname{Rel}\left(F_{i}\right)(R)(u, v) & \text { if } i=j \\ \perp & \text { otherwise }\end{cases}
$$

for $i, j \in I, u \in F_{i} X$ and $v \in F_{j} Y$.

It follows immediately from the above definition that $q \circ \operatorname{Rel}(F)=(F \times F) \circ q$.

Remark 4.3. A general definition of relation lifting, which applies to arbitrary functors on Set, is outside the scope of this paper. Future work will investigate the relevance of the results in $[9,10]$ to this question. The work in loc. cit. shows how to construct truth-preserving predicate liftings and equality-preserving relation liftings for arbitrary functors on the base category of a Lawvere fibration, to the total category of that fibration.

For the remainder of this paper, we take $(S,+, 0, \bullet, 1)$ to be the partial semiring derived in Section 3 from a commutative, partially additive monad T, and we refer to the elements of $S$ as truth values. In the case of the powerset monad, this corresponds to the standard view of relations as subsets, whereas in the case of the sub-probability distribution monad, this results in relations given by valuations in the unit interval.

Example 4.4. Let $F:$ Set $\rightarrow$ Set be given by $F X=1+A \times X$, with $A$ a set (of labels).

- For $\mathrm{\top}=\mathcal{P}, \operatorname{Rel}(F)$ takes a (standard) relation $R \subseteq X \times Y$ to the relation

$$
\left\{\left(\iota_{1}(*), \iota_{1}(*)\right\} \cup\{((a, x),(a, y)) \mid a \in A,(x, y) \in R\} .\right.
$$

- For $\mathrm{\top}=\mathrm{T}_{W}, \operatorname{Rel}(F)$ takes $R: X \times Y \rightarrow \mathbb{N}^{\infty}$ to the relation $R^{\prime}: F X \times F Y \rightarrow \mathbb{N}^{\infty}$ given by

$$
R^{\prime}\left(\iota_{1}(*), \iota_{1}(*)\right)=0 \quad R^{\prime}((a, x),(a, y))=R(x, y) \quad R^{\prime}(u, v)=\infty \text { in all other cases }
$$

- For $\mathrm{T}=\mathcal{S}, \operatorname{Rel}(F)$ takes $R: X \times Y \rightarrow[0,1]$ to the relation $R^{\prime}: F X \times F Y \rightarrow[0,1]$ given by

$$
R^{\prime}\left(\iota_{1}(*), \iota_{1}(*)\right)=1 \quad R^{\prime}((a, x),(a, y))=R(x, y) \quad R^{\prime}(u, v)=0 \text { in all other cases }
$$

- For $\mathrm{T}=\mathcal{L}$, the definition is similar to the case $\mathrm{T}=\mathcal{S}$. 


\section{From Bisimulation to Traces}

Throughout this section we fix a commutative, partially additive monad T : Set $\rightarrow$ Set and assume, as in the previous section, that the natural preorder $\sqsubseteq$ induced by the partial commutative semiring obtained in Section 3 has the multiplication unit $\eta_{1}(*) \in \mathrm{T} 1$ as top element. Furthermore, we assume that this preorder is an $\omega^{\mathrm{op}}$-chain complete partial order, where $\omega^{\mathrm{op}}$-chain completeness amounts to any decreasing chain $x_{1} \sqsupseteq x_{2} \sqsupseteq \ldots$ having a greatest lower bound $\sqcap_{i \in \omega} x_{i}$. These assumptions are clearly satisfied by the preorders in Example 4.1.

We now show how combining the liftings of polynomial functors to the category of generalised relations valued in the partial semiring T1 (as defined in Section 4) with so-called extension liftings arising canonically from the monad T (see Definition 5.2), can be used to give an account of the lineartime behaviour of a state in a coalgebra with branching. The type of such a coalgebra can be any composition involving polynomial endofunctors and the branching monad $\mathrm{T}$, although compositions of type $\mathrm{T} \circ F, G \circ \mathrm{T}$ and $G \circ \mathrm{T} \circ F$ with $F$ and $G$ polynomial endofunctors are particularly emphasised in what follows.

We begin with some informal motivation. When Rel is the standard category of binary relations, recall from Section 2.2 that an $F$-bisimulation is simply a $\operatorname{Rel}(F)$-coalgebra, and that the largest $F$ bisimulation between two $F$-coalgebras $(C, \gamma)$ and $(D, \delta)$ can be obtained as the greatest fixpoint of the monotone operator on $\operatorname{Rel}_{C \times D}$ which takes a relation $R$ to the relation $(\gamma \times \delta)^{*}(\operatorname{Rel}(F)(R))$. Generalising the notion of $F$-bisimulation from standard relations to T1-valued relations makes little sense when the systems of interest are $F$-coalgebras. However, when considering say, coalgebras of type $\mathrm{T} \circ F$, it turns out that liftings of $F$ to the category of T1-valued relations (as defined in Section 4) can be used to describe the linear-time behaviour of states in such a coalgebra, when combined with the previouslymentioned extension liftings. To see why, let us consider labelled transition systems viewed as coalgebras of type $\mathcal{P} \circ(1+A \times \mathrm{Id})$. In such a coalgebra $\gamma: C \rightarrow \mathcal{P}(1+A \times C)$, explicit termination is modelled via transitions $c \rightarrow \iota_{1}(*)$, whereas deadlock (absence of a transition) is modelled as $\gamma(c)=\emptyset$. In this case, $\operatorname{Rel}(\mathcal{P}) \circ \operatorname{Rel}(1+A \times \mathrm{Id})$ is naturally isomorphic to $\operatorname{Rel}(\mathcal{P} \circ(1+A \times \mathrm{Id}))^{4}$, and takes a relation $R \subseteq X \times Y$ to the relation $R^{\prime} \subseteq \mathcal{P}(1+A \times X) \times \mathcal{P}(1+A \times Y)$ given by

$(U, V) \in R^{\prime}$ if and only if $\left\{\begin{array}{l}\text { if } \iota_{1}(*) \in U \text { then } \iota_{1}(*) \in V, \text { and conversely, } \\ \text { if }(a, x) \in U \text { then there exists }(a, y) \in V \text { with }(x, y) \in R, \text { and conversely. }\end{array}\right.$

Thus, the largest $\mathcal{P} \circ(1+A \times \mathrm{Id})$-bisimulation between two coalgebras $(C, \gamma)$ and $(D, \delta)$ can be computed as the greatest fixpoint of the operator on $\operatorname{Rel}_{C, D}$ obtained as the composition

$$
R \subseteq C \times D \stackrel{\operatorname{Rel}(F)}{\longmapsto} R_{1} \subseteq F C \times F D \stackrel{\operatorname{Rel}(\mathcal{P})}{\longmapsto} R_{2} \subseteq \mathcal{P} F C \times \mathcal{P} F D \stackrel{(\gamma \times \delta)^{*}}{\longmapsto} R^{\prime} \subseteq C \times D
$$

where $F=1+A \times$ Id. Recall from Section 2.1 that $\operatorname{Rel}(\mathcal{P})$ takes a relation $R \subseteq X \times Y$ to the relation $R^{\prime} \subseteq \mathcal{P} X \times \mathcal{P} Y$ given by

$(U, V) \in R^{\prime}$ if and only if for all $x \in U$, there exists $y \in V$ such that $(x, y) \in R$, and conversely.

${ }^{4}$ A similar observation holds more generally for $\mathcal{P} \circ F$ with $F$ a polynomial endofunctor. In general, only a natural transformation $\operatorname{Rel}(F \circ G) \Rightarrow \operatorname{Rel}(F) \circ \operatorname{Rel}(G)$ exists, see [15, Exercise 4.4.6]. 
Now consider the effect of replacing $\operatorname{Rel}(\mathcal{P})$ in (2) with the lifting $L:$ Rel $\rightarrow$ Rel that takes a relation $R \subseteq X \times Y$ to the relation $R^{\prime} \subseteq \mathcal{P} X \times Y$ given by

$$
(U, y) \in R^{\prime} \text { if and only if there exists } x \in U \text { with }(x, y) \in R .
$$

To do so, we must change the type of the coalgebra $(D, \delta)$ from $\mathcal{P} \circ F$ to just $F$. A closer look at the resulting operator on $\operatorname{Rel}_{C, D}$ reveals that it can be used to test for the existence of a matching trace: each state of the $F$-coalgebra $(D, \delta)$ can be associated a maximal trace, i.e. an element of the final $F$-coalgebra, by finality. In particular, when $F=1+A \times \mathrm{Id}$, maximal traces are either finite or infinite sequences of elements of $A$. Thus, the greatest fixpoint of the newly defined operator on $\operatorname{Re}_{C \times D}$ corresponds to the relation on $C \times D$ given by

$c \ni_{\mathrm{tr}} d$ if and only if there exists a sequence of choices of transitions starting from $c \in C$ that leads to exactly the same maximal trace (element of $A^{*} \cup A^{\omega}$ ) as the single trace of $d \in D$.

This relation models the ability of the state $c$ to exhibit the same trace as that associated to $d$.

The remainder of this section formalises the above intuitions, and generalises them to arbitrary monads $T$ and polynomial endofunctors $F$, as well as to arbitrary compositions involving the monad $\mathrm{T}$ and several polynomial endofunctors. We begin by restricting attention to coalgebras of type $T \circ F$, with the monad T capturing branching and the endofunctor $F$ describing the structure of individual transitions. In this case it is natural to take the final $F$-coalgebra as the domain of all observable linear-time behaviours of states in $\mathrm{T} \circ F$-coalgebras. Similarly to the above, we let $(C, \gamma)$ and $(D, \delta)$ denote a $\mathrm{T} \circ F$-coalgebra and respectively an $F$-coalgebra. The lifting of $F$ to T1-valued relations will be used as part of a monotone operator on $\operatorname{Rel}_{C, D}$. In order to generalise the lifting $L$ above to arbitrary monads $\mathrm{T}$, we recall the following result from [20], which assumes a strong monad $\mathrm{T}$ on a cartesian closed category.

\section{Proposition 5.1. ([20, Proposition 4.1])}

Let $(B, \beta)$ be a T-algebra. For any $f: X \times Y \rightarrow B$, there exists a unique 1-linear $\bar{f}: \mathrm{T} X \times Y \rightarrow B$ making the following triangle commute:

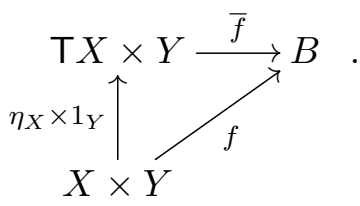

In the above, 1-linearity is linearity in the first variable, assuming the free $\mathrm{T}$-algebra structure on $\mathrm{T} X$ given by $\mu_{X}$. More generally, for T-algebras $(A, \alpha)$ and $(B, \beta)$, a map $f: A \times Y \rightarrow B$ is called 1-linear if the following diagram commutes:



Clearly 1-linearity should be expected of the lifting $L R: \mathrm{T} X \times Y \rightarrow \mathrm{T} 1$ of a relation $R: X \times Y \rightarrow \mathrm{T} 1$, as this amounts to $L R$ commuting with the $\mathrm{T}$-algebra structures $\left(\mathrm{T} X, \mu_{X}\right)$ and $\left(\mathrm{T} 1, \mu_{1}\right)$. Given this, the diagram of Proposition 5.1 forces the definition of the generalised lifting. 


\section{Definition 5.2. (Extension lifting)}

The extension lifting $L_{\mathrm{T}}:$ Rel $\rightarrow$ Rel is the functor taking a relation $R: X \times Y \rightarrow \mathrm{T} 1$ to its unique 1-linear extension $\bar{R}: \mathrm{T} X \times Y \rightarrow \mathrm{T} 1$.

Remark 5.3. It follows from results in [20] that a direct definition of the relation $\bar{R}: \mathrm{T} X \times Y \rightarrow \mathrm{T} 1$ is as the composition

$$
\mathrm{T} X \times Y \stackrel{\mathrm{st}_{X, Y}^{\prime}}{\longrightarrow} \mathrm{T}(X \times Y) \stackrel{\mathrm{T} R}{\longrightarrow} \mathrm{T}^{2} 1 \stackrel{\mu_{1}}{\longrightarrow} \mathrm{T} 1
$$

This also yields functoriality of $L_{\mathrm{T}}$, which follows from the functoriality of its restriction to each fibre category $\operatorname{Rel}_{X, Y}$, as proved next.

Proposition 5.4. The mapping $R \in \operatorname{Rel}_{X, Y} \mapsto \bar{R} \in \operatorname{Rel}_{\mathrm{T} X, Y}$ is functorial.

Proof:

Let $R, R^{\prime} \in \operatorname{Rel}_{X, Y}$ be such that $R \sqsubseteq R^{\prime}$. Hence, there exists $S \in \operatorname{Rel}_{X, Y}$ such that $R+S=R^{\prime}$ (pointwise). Using Remark 5.3, to show that $\bar{R} \sqsubseteq \overline{R^{\prime}}$, it suffices to show that $\mu_{1} \circ \mathrm{T} R \sqsubseteq \mu_{1} \circ \mathrm{T} R^{\prime}$ (pointwise). To this end, we note that commutativity of the map $\delta_{1,1}$ with the monad multiplication, proved in [6, Lemma 15 (iii)] and captured by the commutativity of the lower diagram below (via the solid lines)

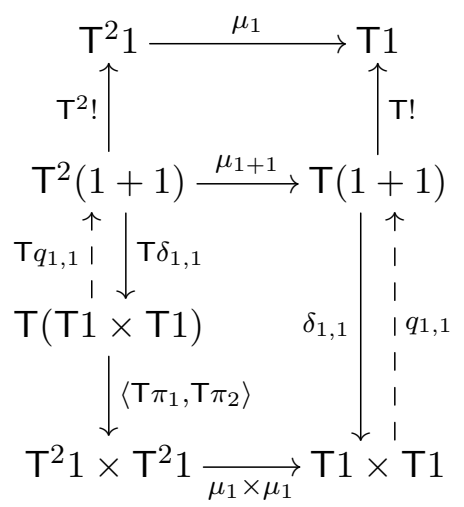

also yields commutativity of the whole diagram (via the dashed arrows). This formalises the commutativity of + (defined as $\mathrm{T} ! \circ q_{1,1}$ ) with the monad multiplication. Now pre-composing this commutative diagram (dashed arrows) with the map

$$
\mathrm{T}(X \times Y) \longrightarrow \mathrm{T}(\mathrm{T} 1 \times \mathrm{T} 1)
$$

given by the image under $\mathrm{T}$ of the map $(x, y) \mapsto\langle R(x, y), S(x, y)\rangle$ yields

$$
\left(\mu_{1} \circ \mathrm{T} R\right)+\left(\mu_{1} \circ \mathrm{T} S\right)=\mu_{1} \circ \mathrm{T}(R+S)=\mu_{1} \circ \mathrm{T} R^{\prime}
$$

and therefore, using the definition of $\sqsubseteq, \mu_{1} \circ \mathrm{T} R \sqsubseteq \mu_{1} \circ \mathrm{T} R^{\prime}$. This concludes the proof.

Thus, $L_{\mathrm{T}}$ is a functor making the following diagram commute:

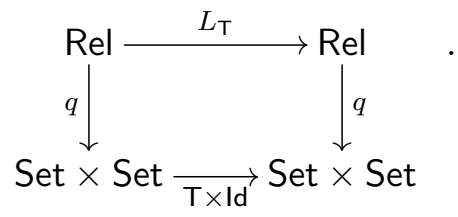


The (dual of the) following standard result concerning the existence of least fixpoints of monotone operators on chain-complete partial orders will now be used to define linear-time behaviour.

\section{Theorem 5.5. ([7, Theorem 8.22])}

Let $P$ be a complete partial order and let $\mathcal{O}: P \rightarrow P$ be order-preserving. Then $\mathcal{O}$ has a least fixpoint.

We are now ready to give an alternative account of maximal traces of (states in) $T \circ F$-coalgebras.

\section{Definition 5.6. (Maximal trace map)}

Let $(C, \gamma)$ denote a $\mathrm{T} \circ F$-coalgebra, and let $(Z, \zeta)$ denote the final $F$-coalgebra. The maximal trace map $\operatorname{tr}_{\gamma}: C \rightarrow(\mathrm{T} 1)^{Z}$ of $\gamma$ is the exponential transpose of the greatest fixpoint $R: C \times Z \rightarrow \mathrm{T} 1$ of the operator $\mathcal{O}: \operatorname{Rel}_{C, Z} \rightarrow \operatorname{Rel}_{C, Z}$ given by the composition

$$
\operatorname{Rel}_{C, Z} \stackrel{\operatorname{Rel}(F)}{\longrightarrow} \operatorname{Rel}_{F C, F Z} \stackrel{L_{\mathrm{T}}}{\longrightarrow} \operatorname{Rel}_{\mathrm{T} F C, F Z} \stackrel{(\gamma \times \zeta)^{*}}{\longrightarrow} \operatorname{Rel}_{C, Z}
$$

Definition 5.6 makes use of Theorem 5.5 applied to the dual of the order $\sqsubseteq$. Our assumption that $\sqsubseteq$ is $\omega^{\mathrm{op}}$-chain complete makes the dual order a chain-complete partial order. Monotonicity of the operator in Definition 5.6 is an immediate consequence of the functoriality of $\operatorname{Rel}(F), L_{\mathrm{T}}$ and $(\gamma \times \delta)^{*}$.

A construction for the least fixpoint of an order-preserving operator on a complete partial order, which involves taking a limit over an ordinal-indexed chain, is also described in [7]. Instantiating this construction to the dual of the order $\sqsubseteq$ yields an ordinal-indexed sequence of relations $\left(R_{\alpha}\right)$, where:

- $R_{0}=\top$ (i.e. the relation on $C \times Z$ given by $(c, z) \mapsto 1$ ),

- $R_{\alpha+1}=\mathcal{O}\left(R_{\alpha}\right)$,

- $R_{\alpha}=\Pi_{\beta<\alpha} R_{\beta}$, if $\alpha$ is a limit ordinal.

The greatest fixpoint of the operator $\mathcal{O}$ is the limit of this sequence.

Example 5.7. Let $F$ denote an arbitrary polynomial functor (e.g. $1+A \times \mathrm{Id}$ ).

- For $T=\mathcal{P}$, the extension lifting $L_{\mathcal{P}}:$ Rel $\rightarrow$ Rel takes a (standard) relation $R \subseteq X \times Y$ to the relation $L_{\mathcal{P}} R \subseteq \mathcal{P} X \times Y$ given by

$$
(U, y) \in L_{\mathcal{P}} R \text { if and only if there exists } x \in U \text { with }(x, y) \in R
$$

for $U \in \mathcal{P} X$ and $y \in Y$. As a result, the greatest fixpoint of the operator $\mathcal{O}$ from Definition 5.6 relates a state $c$ in a $\mathcal{P} \circ F$-coalgebra $(C, \gamma)$ with a state $z$ of the final $F$-coalgebra if and only if there exists a sequence of choices in the unfolding of $\gamma$ starting from $c$, that results in an $F$ behaviour bisimilar to $z$. This was made more precise in [2], where infinite two-player games were developed for verifying whether a state of a $\mathcal{P} \circ F$-coalgebra can exhibit a certain maximal trace (element of the final $F$-coalgebra).

- For $\mathrm{T}=\mathrm{T}_{W}$, the extension lifting $L_{W}:$ Rel $\rightarrow$ Rel takes a weighted relation $R: X \times Y \rightarrow W$ to the relation $L_{W} R: \mathrm{T}_{W} X \times Y \rightarrow W$ given by

$$
\left(L_{W} R\right)(\varphi, y)=\min _{x \in \sup (\varphi)}(\varphi(x)+R(x, y))
$$


for $\varphi: X \rightarrow W$ and $y \in Y$. Thus, the greatest fixpoint of $\mathcal{O}$ maps a pair $(c, z)$, with $c$ a state in a $\mathrm{T}_{W} \circ F$-coalgebra and $z$ a maximal trace, to the cost (computed via the min function) of exhibiting that trace. The computation of the fixpoint starts from a relation that maps each pair of states $(c, z)$ to the value $0 \in \mathbb{N}^{\infty}$ (the top element for $\sqsubseteq$ ), and refines this down (w.r.t. the $\sqsubseteq$ order) through step-wise unfolding of the coalgebra structures $\gamma$ and $\zeta$.

- For $T=T_{\mathcal{S}}$, the extension lifting $L_{\mathcal{S}}:$ Rel $\rightarrow$ Rel takes a valuation $R: X \times Y \rightarrow[0,1]$ to the valuation $L_{\mathcal{S}} R: \mathcal{S} X \times Y \rightarrow[0,1]$ given by

$$
\left(L_{\mathcal{S}} R\right)(\varphi, y)=\sum_{x \in \sup (\varphi)} \varphi(x) * R(x, y)
$$

for $\varphi \in \mathcal{S} X$ and $y \in Y$. Thus, the greatest fixpoint of $\mathcal{O}$ yields, for each state in a $\mathcal{S} \circ F$-coalgebra and each potential maximal trace, the probability of this trace being exhibited from that state. As computing these probabilities amounts to multiplying possibly infinitely-many probability values, the probability of an infinite trace will often be 0 (unless from some point in the unfolding of a particular state, probability values of 1 are associated to the individual transitions that match a particular infinite trace). This may appear as a deficiency of our framework, and one could argue that a measure-theoretic approach, whereby a probability measure is derived from the probabilities of finite prefixes of infinite traces, would be more appropriate. However, we remark that an extension of the present approach to linear-time logics, where individual maximal traces are replaced by linear-time temporal logic formulas, does not suffer from this deficiency [4]. In particular, lineartime formulas involving least fixpoints can be used to represent finite maximal traces, or sets of such traces.

- For $T=\mathcal{L}$, the extension lifting $L_{\mathcal{L}}:$ Rel $\rightarrow$ Rel takes a relation $R: X \times Y \rightarrow\{0,1\}$ to the relation $\left(L_{\mathcal{L}} R\right): \mathcal{L} X \times Y \rightarrow\{0,1\}$ given by

$$
\left(L_{\mathcal{L}} R\right)\left(\iota_{1}(\perp), y\right)=0 \quad\left(L_{\mathcal{L}} R\right)\left(\iota_{2}(x), y\right)=R(x, y)
$$

for $x \in X$ and $y \in Y$. Thus, the greatest fixpoint of $\mathcal{O}$ yields, for each state in a $\mathcal{L} \circ F$-coalgebra and each potential maximal trace, a value of 1 precisely when that trace is exhibited from the given state.

Example 5.8. Consider again the two transition systems from Figure 1, viewed as $\mathcal{P} \circ(1+\mathrm{ld})$-coalgebras:
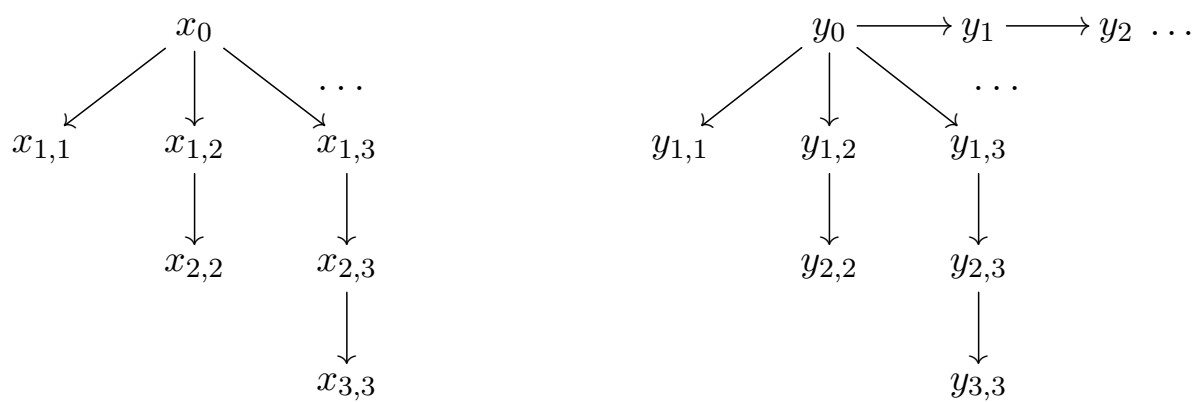

The final $1+$ Id-coalgebra has carrier (isomorphic to) $\mathbb{N}^{\infty}$, and its coalgebra map takes 0 to $\iota_{1}(*)$, $n+1$ to $\iota_{2}(n)$ for $n=0,1, \ldots$ and $\infty$ to $\iota_{2}(\infty)$. In this example, $R_{\alpha+1}\left(x_{0}, \infty\right)=R_{\alpha+1}\left(y_{0}, \infty\right)=1$ 
for each $\alpha<\omega$ (since, for each such $\alpha$, we have $R_{\alpha}\left(x_{1, \alpha+1}, \infty\right)=R_{\alpha}\left(y_{1, \alpha+1}, \infty\right)=1$ ), however, $R_{\omega}\left(x_{0}, \infty\right)=0$ (since for each $\alpha<\omega, R_{\alpha+1}\left(x_{1, \alpha+1}, \infty\right)=0$ ), whereas $R_{\omega}\left(y_{0}, \infty\right)=1$ (since $R_{\alpha}\left(y_{n}, \infty\right)=1$ for each $\alpha<\omega$ and each $\left.n \in\{0,1, \ldots\}\right)$. As a result, the maximal trace map of Definition 5.6 distinguishes the states $x_{0}$ and $y_{0}$, as claimed earlier.

The next remark highlights the generality of our approach, by showing that it can also be used to derive finite traces, and at the same time has a potential application to verification.

Remark 5.9. By replacing the $F$-coalgebra $(Z, \zeta)$ by $\left(I, \alpha^{-1}\right)$ with $(I, \alpha)$ an initial $F$-algebra, one obtains an alternative account of finite traces of states in $T \circ F$-coalgebras, with the finite trace map $\mathrm{ftr}_{\gamma}: C \rightarrow(\mathrm{T} 1)^{I}$ of a $\mathrm{T} \circ F$-coalgebra $(C, \gamma)$ being obtained via the greatest fixpoint of essentially the same operator $\mathcal{O}$, but this time on $\operatorname{Rel}_{C, I}$. In fact, one can use any $F$-coalgebra in place of $(Z, \zeta)$. For verification purposes, where one is typically interested in a specific linear-time behaviour (e.g. capturing an undesirable property), a coalgebra with a finite state space, encoding that particular behaviour, often suffices. For example, to verify that a non-deterministic system modelled as a $\mathcal{P} \circ(1+$ Id $)$-coalgebra always terminates, one can use the (single-state) $1+$ Id-coalgebra $\left(1, \iota_{2}\right)$, whose only state encodes non-termination, to check for the existence of a non-terminating behaviour.

Remark 5.10. It follows from standard fixpoint theory (see e.g. [25, Theorem 2.12]) that for $\mathrm{T}=\mathcal{P}$ (in which case $\operatorname{Rel}_{C, Z}$ is complete lattice), restricting to a finite-state $\mathcal{P} \circ F$-coalgebra $(C, \gamma)$ and a finitestate $F$-coalgebra $(D, \delta)$ results in the greatest fixpoint of $\mathcal{O}$ being reached in a finite number of steps. However, for $\mathrm{T}=\mathcal{S}$ or $T=\mathrm{T}_{W}$, this is generally not the case. To see this, it suffices to consider the following two-state $\mathcal{S}$ - and respectively $\mathrm{T}_{W}$-coalgebras:
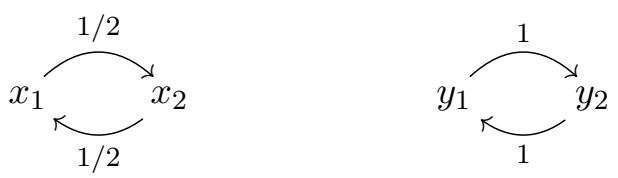

and observe that, while in both cases the domain of linear-time behaviours is the singleton set $1=\{*\}$ (as $F=\mathrm{Id}$ ), the fixpoint only stabilises at $\omega$ (to $\left(x_{i}, *\right) \mapsto 0$, respectively $\left(y_{i}, *\right) \mapsto \infty$, for $i=1,2$ ). However, for probabilistic or weighted computations, an approximation of the greatest fixpoint may be sufficient in practice, as thresholds can be provided as part of specific verification tasks. For example, in a probabilistic system, a guarantee that an error behaviour occurs with probability below a given threshold is often sufficient. Similarly, in weighted computations, a proof that the minimal cost of achieving a certain linear-time behaviour is larger than an acceptable threshold already indicates a problem.

Remark 5.11. The choice of functor $F$ directly impacts on the notion of linear-time behaviour. For example, by regarding labelled transition systems as coalgebras of type $\mathcal{P} \circ(A \times \mathrm{Id})$ instead of $\mathcal{P} \circ(1+$ $A \times$ Id) (i.e. not modelling successful termination explicitly), finite traces are not anymore accounted for, as the elements of the final $F$-coalgebra are now given by infinite sequences of elements of $A$. This should not be regarded as a drawback, in fact it further illustrates the flexibility of our approach, as the functor $F$ can be chosen so as to match an expected notion of linear-time behaviour. We will return to this observation in Example 5.12. 
The approach presented above also applies to coalgebras of type $G \circ \mathrm{T}$ with $G$ a polynomial endofunctor, and more generally to coalgebras whose type is obtained as the composition of polynomial endofunctors and the monad $\mathrm{T}$, with possibly several occurrences of $\mathrm{T}$ in this composition. In the case of $G \circ$ T-coalgebras, instantiating our approach yields different results to the extension semantics proposed in [16]. Specifically, the instantiation involves taking $(Z, \zeta)$ to be a final $G$-coalgebra and $(C, \gamma)$ to be an arbitrary $G \circ \mathrm{T}$-coalgebra, and considering the monotone operator on $\operatorname{Rel}_{C, Z}$ given by the composition

$$
\operatorname{Rel}_{C, Z} \stackrel{L_{\mathrm{T}}}{\longrightarrow} \operatorname{Rel}_{\mathrm{T} C, Z} \stackrel{\operatorname{Rel}(G)}{\longrightarrow} \operatorname{Rel}_{G(\mathrm{~T} C), G Z} \stackrel{(\gamma \times \zeta)^{*}}{\longrightarrow} \operatorname{Rel}_{C, Z}
$$

The following example illustrates the difference between our approach and that of [16].

Example 5.12. For $G=2 \times \mathrm{Id}^{A}$ with 2 a two-element set, $A$ a finite alphabet and $\mathrm{T}=\mathcal{P}, G \circ \mathrm{T}$ coalgebras are non-deterministic automata, whereas the elements of the final $G$-coalgebra, given by functions $z: A^{*} \rightarrow 2$, correspond to languages over $A$. In this case, the greatest fixpoint of the operator in (3) maps a pair $(c, z)$, with $c$ a state of a non-deterministic automaton and $z$ a language over $A$, to $\top$ if and only if there exists a sequence of choices in the unfolding of the automaton starting from $c$ that results in precisely the words in $z$ being accepted - but now by a "machine" that does not anymore have a finite number of states, given the unfolding of the original automaton. This is different from the approach of [16], which precisely matches the notion of language acceptance by a non-deterministic automaton. In our approach, taking the union of all $z \mathrm{~s}$ (viewed as languages over $A$ ) such that $(c, z)$ is mapped to $\top$ recovers the language accepted by the non-deterministic automaton with $c$ as initial state, but only under the assumption that for each $a \in A$, an $a$-labelled transition exists from any state of the automaton. At the root of this difference w.r.t. [16] lies our choice (reflected in the use of the functor $G$ ) to take, as notion of linear-time behaviour, an entire language over $A$ (as opposed to a single word over $A$ ). If non-deterministic automata were instead modelled as $\mathcal{P} \circ(1+A \times \mathrm{Id})$-coalgebras (where we note the isomorphism $\left.\mathcal{P} \circ(1+A \times \mathrm{Id}) \simeq \mathcal{P} 1 \times \mathcal{P}^{A}\right)$, this would correspond to linear-time behaviours (elements of the final $1+A \times$ Id-coalgebra) being given by finite or infinite sequences of elements of $A$. This time, by restricting attention to finite linear-time behaviours (elements of the initial $1+A \times$ Id-coalgebra, which correspond to words over $A$ ), one recovers the familiar automata-theoretic notion of acceptance of a word: the greatest fixpoint of the operator $\mathcal{O}$ of Definition 5.6 relates a state $c$ of a $\mathcal{P} \circ(1+A \times \mathrm{Id})$ coalgebra with a finite behaviour $a_{1} \ldots a_{n} *$ if and only if the associated automaton (with initial state $c$ ) accepts the word $a_{1} \ldots a_{n}$.

Finally, we consider the general case of coalgebras whose type is obtained as the composition of several endofunctors on Set, one of which is a monad $\mathrm{T}$ that accounts for the presence of branching in the system, while the remaining endofunctors are polynomial and jointly determine the notion of linear-time behaviour. For simplicity of presentation, we only consider coalgebras of type $G \circ \mathrm{T} \circ F$, with the final $G \circ F$-coalgebra $(Z, \zeta)$ providing the domain of possible linear-time behaviours.

\section{Definition 5.13. (Linear-time behaviour)}

The linear-time behaviour of a state in a coalgebra $(C, \gamma)$ of type $G \circ \mathrm{T} \circ F$ is the greatest fixpoint of the operator $\mathcal{O}$ on $\operatorname{Rel}_{C, Z}$ defined by the composition:

$$
\operatorname{Rel}_{C, Z} \stackrel{\operatorname{Rel}(F)}{\longrightarrow} \operatorname{Rel}_{F C, F Z} \stackrel{L_{\mathrm{T}}}{\longrightarrow} \operatorname{Rel}_{\mathrm{T} F C, F Z} \stackrel{\operatorname{Rel}(G)}{\longrightarrow} \operatorname{Rel}_{G \mathrm{~T} F C, G F Z} \stackrel{(\gamma \times \zeta)^{*}}{\longrightarrow} \operatorname{Rel}_{C, Z}
$$


The greatest fixpoint of $\mathcal{O}$ measures the extent to which a state in a $G \circ \mathrm{T} \circ F$-coalgebra can exhibit a given linear-time behaviour (element of the final $G \circ F$-coalgebra).

Example 5.14. Coalgebras of type $G \circ \mathrm{T} \circ F$, where $G=\mathrm{Id}^{A}$ and $F=1+B \times \mathrm{Id}$, model systems with branching, with both inputs (from a finite set $A$ ) and outputs (in a set $B$ ). In this case, the possible linear-time behaviours (elements of the final $G \circ F$-coalgebra) are given by special trees, with both finite and infinite branches, whose edges are labelled by elements of $A$ (one outgoing edge from each node for each $a \in A$ ), and whose nodes (with the exception of the root, which is not labelled) are either labelled by $* \in 1$ (in the case of leaves) or by an element of $B$ (in the case of non-leaves). The linear-time behaviour of a state in a $G \circ \mathrm{T} \circ F$-coalgebra then gives:

- the set of such trees that can be exhibited from that state, when $T=\mathcal{P}$,

- the minimum cost of exhibiting each tree (with the costs associated to different branches of the tree being added when computing this cost), when $\mathrm{T}=\mathrm{T}_{W}$,

- the probability of exhibiting each such tree (with the probabilities corresponding to different tree branches being multiplied when computing this probability), when $\mathrm{T}=\mathcal{S}$, and

- a value of 1 if and only if such a tree can be exhibited, when $T=\mathcal{L}$.

In the case of probabilistic systems, the intuition is that in order to match a particular tree, one needs to match the expected behaviour for every possible sequence of inputs, and therefore multiplying probabilities associated to different tree branches (different inputs) is the right choice. Similarly, in the case of weighted systems, the cost of matching a particular tree must take into account the cost of matching the specified behaviour for each branch of this tree, and thus taking the sum of the costs associated to different tree branches (different inputs) gives again the expected result.

Remark 5.15. Our approach does not directly apply to the probability distribution monad (defined similarly to the sub-probability distribution monad, but with probabilities adding up to exactly 1 ), as this monad does not satisfy the condition $T \emptyset=1$ of Definition 3.2. However, systems where branching is described using probability distributions can still be dealt with, by regarding all probability distributions as sub-probability distributions.

Definition 5.13 generalises straightforwardly to coalgebraic types given by arbitrary compositions of polynomial endofunctors and the monad $\mathrm{T}$, with the extension lifting $L_{\mathrm{T}}$ being used once for each occurrence of $\mathrm{T}$ in such a composition. However, we currently do not have interesting examples for the case when the monad T occurs twice in the coalgebraic type. Indeed, in such cases, one may want to treat the two occurrences of T differently, and view only one of these occurrences as specifying branching in the same way in which, when two different monads appear in the coalgebraic type, one typically chooses one of the monads as the branching type. Our final example illustrates such a situation, by showing how to extend the definition of linear-time behaviour to coalgebras whose type does not satisfy the assumptions of Definition 5.13. While not canonical anymore, the resulting notion of linear-time behaviour can encompass an even wider, and arguably more practically-relevant class of systems with branching. 
Example 5.16. Coalgebras of type $\mathcal{P} \circ \mathcal{S} \circ(A \times \mathrm{Id})$, also known as Segala systems, are models of systems incorporating both non-deterministic and probabilistic branching. Such models are used e.g. in the verification of concurrent probabilistic programs, where one needs to consider the interaction between probabilistic components and non-deterministic schedulers, or in the verification of probabilistic security protocols, where the unknown environment is modelled non-deterministically. Our approach can be adapted to accommodate such coalgebras in the following way: the monad $\mathcal{S}$ is chosen as the branching monad (resulting in the unit interval as the universe of truth values), the polynomial endofunctor $A \times \mathrm{Id}$ is used to define the notion of trace, and finally a relation lifting $\tilde{L}_{\mathcal{P}}: \operatorname{Rel} \rightarrow \operatorname{Rel}$ of the functor $\mathcal{P} \times \operatorname{Id}$ is used to abstract away (to a certain degree) the non-deterministic branching. The corresponding operator on Rel is thus obtained as the composition:

$$
\operatorname{Rel}_{C, Z} \stackrel{\operatorname{Rel}(A \times \mathrm{Id})}{\longrightarrow} \operatorname{Rel}_{A \times C, A \times Z} \stackrel{L_{\mathcal{S}}}{\longrightarrow} \operatorname{Rel}_{\mathcal{S}(A \times C), A \times Z} \stackrel{\tilde{L}_{\mathcal{P}}}{\longrightarrow} \operatorname{Rel}_{\mathcal{P S}(A \times C), A \times Z} \stackrel{(\gamma \times \zeta)^{*}}{\longrightarrow} \operatorname{Rel}_{C, Z}
$$

where the lifting $\tilde{L}_{\mathcal{P}}$ takes $R: X \times Y \rightarrow[0,1]$ to the relation $\tilde{L}_{\mathcal{P}} R: \mathcal{P} X \times Y \rightarrow[0,1]$ given by

$$
\left(\tilde{L}_{\mathcal{P}} R\right)(U, y)=\inf _{x \in U} R(x, y)
$$

for $U \in \mathcal{P} X$ and $y \in Y$. (A definition which replaces inf by sup is equally possible.) An alternative definition of $\tilde{L}_{\mathcal{P}} R$ is as the composition

$$
\mathcal{P} X \times Y \stackrel{\mathrm{st}_{X, Y}^{\prime}}{\longrightarrow} \mathcal{P}(X \times Y) \stackrel{\mathcal{P} R}{\longrightarrow} \mathcal{P} \mathcal{S} 1 \stackrel{\alpha}{\longrightarrow} \mathcal{S} 1
$$

with $\alpha: \mathcal{P S} 1 \rightarrow \mathcal{S} 1$ taking a set of probability values to its infimum (respectively supremum). We note the similarity between this latter formulation and the definition of $L_{\mathrm{T}}$, as given in Remark 5.3. At the same time, we note the difference between the types of the relation liftings $\operatorname{Rel}(A \times \mathrm{Id})$ and $\tilde{L}_{\mathcal{P}}$ : the former is used to propagate linear-time behaviour, whereas the latter is used to abstract away non-deterministic branching. The induced notion of linear-time behaviour, defined similarly to Definition 5.13, gives for each state in a Segala system and each maximal trace (infinite sequence of elements of $A$ ), the lowest (respectively highest) probability of that trace being exhibited, taken across all possible scheduling choices/adversaries. The above fixpoint characterisation is similar to the use of value iteration in probabilistic model checking (e.g. model checking the logic PCTL interpreted over Markov decision processes [21]). The use of such an approach to reason about the interaction between components with different computational types (non-deterministic, stochastic, weighted), will be further explored in future work.

It is not difficult to see that the approach outlined in Example 5.16 generalises to arbitrary compositions of (i) a single branching monad, which canonically induces a universe of truth values, (ii) a number of polynomial endofunctors, which jointly induce a notion of trace, and (iii) several other endofunctors, each equipped with a suitable lifting to Rel.

In the remainder of this section, we briefly explore the usefulness of an operator similar to $\mathcal{O}$ of Definition 5.6, which employs an extension lifting arising from the double strength of the monad T. For simplicity of presentation, we do not consider the more general case of Definition 5.13, however, essentially the same analysis can also be carried out for that case.

We begin by noting that a result similar to Proposition 5.1 is proved in [20] for a commutative monad on a cartesian closed category. 
Proposition 5.17. ([20, Proposition 9.3])

Let $(B, \beta)$ be a T-algebra. Then any $f: X \times Y \rightarrow B$ extends uniquely along $\eta_{X} \times \eta_{Y}$ to a bilinear $\tilde{f}: \mathrm{T} X \times \mathrm{T} Y \rightarrow B$, making the following triangle commute:

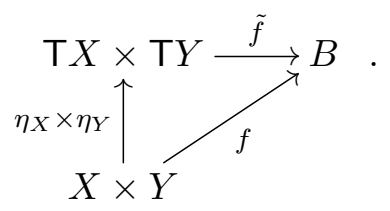

Here, bilinearity amounts to linearity in each argument, again assuming the free $\mathrm{T}$-algebra structures on $\mathrm{T} X$ and $\mathrm{T} Y$ given by $\mu_{X}$ and $\mu_{Y}$, respectively.

\section{Definition 5.18. (Double extension lifting)}

For a commutative monad T : Set $\rightarrow$ Set, the double extension lifting $L_{\mathrm{T}}^{\prime}:$ Rel $\rightarrow$ Rel is the functor taking a relation $R: X \times Y \rightarrow \mathrm{T} 1$ to its unique bilinear extension $\tilde{R}: \mathrm{T} X \times \mathrm{T} Y \rightarrow \mathrm{T} 1$.

Remark 5.19. An alternative definition of $L_{\mathrm{T}}^{\prime}$ is as the composition of $L_{\mathrm{T}}$ with a dual lifting, which takes a relation $R: X \times Y \rightarrow \mathrm{T} 1$ to its unique 2-linear extension $\bar{R}: X \times \mathrm{T} Y \rightarrow \mathrm{T} 1$.

Remark 5.20. Again, it can be shown that a direct definition of the relation $\tilde{R}: \mathrm{T} X \times \mathrm{T} Y \rightarrow \mathrm{T} 1$ is as the composition

$$
\mathrm{T} X \times \mathrm{T} Y \stackrel{\mathrm{dst}_{X, Y}}{\longrightarrow} \mathrm{T}(X \times Y) \stackrel{\mathrm{T} R}{\longrightarrow} \mathrm{T}^{2} 1 \stackrel{\mu_{1}}{\longrightarrow} \mathrm{T} 1
$$

Proposition 5.21. The mapping $R \in \operatorname{Rel}_{X, Y} \mapsto \bar{R} \in \operatorname{Rel}_{X, T Y}$ is functorial.

We now fix two $\mathrm{T} \circ F$-coalgebras $(C, \gamma)$ and $(D, \delta)$ and explore the greatest fixpoint of the operator $\mathcal{O}^{\prime}: \operatorname{Rel}_{C, D} \rightarrow \operatorname{Rel}_{C, D}$ defined by the composition

$$
\operatorname{Rel}_{C, D} \stackrel{\operatorname{Rel}(F)}{\longrightarrow} \operatorname{Rel}_{F C, F D} \stackrel{L_{\mathrm{T}}^{\prime}}{\longrightarrow} \operatorname{Rel}_{\mathrm{T} F C, \mathrm{~T} F D} \stackrel{(\gamma \times \zeta)^{*}}{\longrightarrow} \operatorname{Rel}_{C, D}
$$

As before, the operator $\mathcal{O}^{\prime}$ is monotone and therefore admits a greatest fixpoint. We argue (through the next example) that this fixpoint also yields useful information regarding the linear-time behaviour of states in $T \circ F$-coalgebras. Moreover, this generalises to coalgebras whose types are arbitrary compositions of polynomial functors and the branching monad $\mathrm{T}$.

Example 5.22. Let $F$ : Set $\rightarrow$ Set be a polynomial endofunctor, defining a certain type of linear-time behaviour.

1. For non-deterministic systems $(T=\mathcal{P})$, the double extension lifting $L_{\mathcal{P}}^{\prime}:$ Rel $\rightarrow$ Rel takes a (standard) relation $R \subseteq X \times Y$ to the relation $L_{\mathcal{P}}^{\prime} R \subseteq \mathcal{P} X \times \mathcal{P} Y$ given by

$$
(U, V) \in L_{\mathcal{P}}^{\prime} R \text { if and only if there exist } x \in U \text { and } y \in V \text { with }(x, y) \in R
$$

for $U \in \mathcal{P} X$ and $V \in \mathcal{P} Y$. The greatest fixpoint of $\mathcal{O}^{\prime}$ therefore relates two states of two $\mathcal{P} \circ F$ coalgebras if and only if they admit a common maximal trace. 
2. For weighted systems $\left(\mathrm{T}=\mathrm{T}_{W}\right)$, the lifting $L_{W}^{\prime}:$ Rel $\rightarrow$ Rel takes a weighted relation $R$ : $X \times Y \rightarrow W$ to the relation $L_{W}^{\prime} R: \mathrm{T}_{W} X \times \mathrm{T}_{W} Y \rightarrow W$ given by

$$
\left(L_{W}^{\prime} R\right)(\varphi, \psi)=\min _{x \in \sup (\varphi), y \in \sup (\psi)}(\varphi(x)+\psi(y)+R(x, y))
$$

for $\varphi: X \rightarrow W$ and $\psi: Y \rightarrow W$. As a result, the greatest fixpoint of $\mathcal{O}^{\prime}$ measures the joint minimal cost of two states of two $\mathrm{T}_{W} \circ F$-coalgebras exhibiting the same maximal trace.

3. For probabilistic systems $\left(\mathrm{T}=\mathcal{S}\right.$ ), the lifting $L_{\mathcal{S}}^{\prime}$ is defined similarly to $L_{W}^{\prime}$, but with min replaced by + , and with + replaced by $*$. This time the greatest fixpoint of $\mathcal{O}^{\prime}$ measures the probability of two states exhibiting the same maximal trace.

4. For $\mathrm{T}=\mathcal{L}$, the lifting $L_{\mathcal{L}}^{\prime}: \operatorname{Rel} \rightarrow$ Rel takes a relation $R: X \times Y \rightarrow\{0,1\}$ to the relation $L_{\mathcal{L}}^{\prime} R: \mathcal{L} X \times \mathcal{L} Y \rightarrow\{0,1\}$ given by

$$
\left(L_{\mathcal{L}}^{\prime} R\right)\left(\iota_{2}(x), \iota_{2}(y)\right)=R(x, y) \quad\left(L_{\mathcal{L}}^{\prime} R\right)(u, v)=0 \text { in all other cases }
$$

for $x \in X, y \in Y, u \in \mathcal{L} X$ and $v \in \mathcal{L} Y$. Thus, the greatest fixpoint of $\mathcal{O}^{\prime}$ gives a value of 1 for a pair of states of two $\mathcal{L} \circ F$-coalgebras if and only if the two states have normal (i.e. non-exception) behaviour and they exhibit the same maximal trace.

In all the above cases, the greatest fixpoint of $\mathcal{O}^{\prime}$ can be viewed as measuring the extent of the similarity between states of $T \circ F$-coalgebras. Again, future work will explore the usefulness of such a fixpoint characterisation, particularly for model checking, where one can take one of the coalgebras to define the system to be verified, and the other to specify unacceptable behaviours.

\section{Conclusions and Future Work}

We have provided a general and uniform account of the linear-time behaviour of a state in a coalgebra whose type incorporates some notion of branching (captured by a partially additive monad on Set). Our approach is compositional, and so far applies to notions of linear behaviour specified by polynomial endofunctors on Set. The key ingredient of our approach is the notion of extension lifting, which allows the branching behaviour of a state to be abstracted away in a coinductive fashion.

As emphasised in Remark 5.9, our approach goes beyond linear-time behaviour, also allowing a coalgebraic treatment of finite traces. Future work will investigate the relationship to existing coalgebraic accounts of finite traces $[12,16]$. At this point, we observe that such approaches are dependent on a choice of distributive law, of either the monad T over the endofunctor $F$ (in the case of $T \circ F$-coalgebras), or of the endofunctor $G$ over the monad T (in the case of $G \circ \mathrm{T}$-coalgebras). While our approach does not assume such a distributive law, one can show that for any commutative monad T on Set, a canonical choice of distributive law of T over $F$ exists [5]; its definition makes use of the multiplication operation - on T1. Such a canonical distributive law was used in loc. cit. to study linear time coalgebraic logics with two equivalent semantics: a step-wise semantics similar to that of other coalgebraic modal logics, and a path-based semantics akin to those of standard linear-time logics.

Other future work will attempt to exploit the results of $[9,10]$ in order to define generalised relation liftings for arbitrary endofunctors on Set, and to extend our approach to other base categories (with the 
category of measurable spaces being one of the categories of interest). The work in loc. cit. could also provide an alternative description for the greatest fixpoint used in Definition 5.13.

The present work constitutes a stepping stone towards a coalgebraic approach to the formal verification of linear-time properties. Some initial steps in this direction have already been made in [4], where a similar approach to defining linear-time fixpoint logics was taken. However, further work is needed to study the expressiveness of the proposed logics, as well as suitable verification techniques for these logics. For the latter, an automata-based approach, as proposed in [2] for the case of non-deterministic systems, appears promising.

\section{References}

[1] Cîrstea, C.: Maximal Traces and Path-Based Coalgebraic Temporal Logics, Theor. Comput. Sci., 412(38), 2011, 5025-5042.

[2] Cîrstea, C.: Model Checking Linear Coalgebraic Temporal Logics: An Automata-Theoretic Approach, in: Proceedings CALCO 2011 (A. Corradini, B. Klin, C. Cîrstea, Eds.), vol. 6859 of Lect. Notes Comput. Sci., Springer, 2011, 130-144.

[3] Cîrstea, C.: From Branching to Linear Time, Coalgebraically, in: Proceedings FICS 2013 (D. Baelde, A. Carayol, Eds.), vol. 126 of Electron. Proc. Theor. Comput. Sci., 2013, 11-27.

[4] Cîrstea, C.: A Coalgebraic Approach to Linear-Time Logics, in: Proceedings FOSSACS 2014, vol. 8412 of Lect. Notes Comput. Sci., Springer, 2014, 428-442.

[5] Cîrstea, C.: Canonical Coalgebraic Linear Time Logics, in: Proceedings CALCO 2015 (L. Moss, P. Sobocinski, Eds.), Leibniz International Proceedings in Informatics, Schloss Dagstuhl-Leibniz-Zentrum für Informatik, 2015, To appear.

[6] Coumans, D., Jacobs, B.: Scalars, Monads, and Categories, in: Quantum Physics and Linguistics. A Compositional, Diagrammatic Discourse (C. Heunen, M. Sadrzadeh, E. Grefenstette, Eds.), Oxford Univ. Press, 2013, 184-216.

[7] Davey, B. A., Priestley, H. A.: Introduction to Lattices and Order (2. ed.), Cambridge University Press, 2002.

[8] Ésik, Z., Kuich, W.: Modern Automata Theory, 2007, http://dmg.tuwien.ac.at/kuich/.

[9] Fumex, C., Ghani, N., Johann, P.: Indexed Induction and Coinduction, Fibrationally, in: Proceedings CALCO 2011 (A. Corradini, B. Klin, C. Cîrstea, Eds.), vol. 6859 of Lect. Notes Comput. Sci., Springer, 2011, 176-191.

[10] Ghani, N., Johann, P., Fumex, C.: Generic Fibrational Induction, Log. Methods Comput. Sci., 8(2), 2012.

[11] Hasuo, I., Cho, K., Kataoka, T., Jacobs, B.: Coinductive Predicates and Final Sequences in a Fibration, in: Proceedings MFPS XXIX, vol. 298 of Electr. Notes Theor. Comput. Sci., Elsevier, 2013, 197-214.

[12] Hasuo, I., Jacobs, B., Sokolova, A.: Generic Trace Semantics via Coinduction, Log. Methods Comput. Sci., 3(4), 2007, 1-36.

[13] Hermida, C., Jacobs, B.: Structural Induction and Coinduction in a Fibrational Setting, Inf. Comput., 145(2), 1998, 107-152.

[14] Jacobs, B.: Trace Semantics for Coalgebras, in: Proceedings CMCS 2004, vol. 106 of Electr. Notes Theor. Comput. Sci., Elsevier, 2004, 167-184. 
[15] Jacobs, B.: Introduction to Coalgebra. Towards Mathematics of States and Observations (Version 2.0), 2012, http://www.cs.ru.nl/B. Jacobs/CLG/JacobsCoalgebraIntro.pdf.

[16] Jacobs, B., Silva, A., Sokolova, A.: Trace Semantics via Determinization, in: Proceedings CMCS 2012, vol. 7399 of Lect. Notes Comput. Sci., Springer, 2012, 109-129.

[17] Kanellakis, P. C., Smolka, S. A.: CCS Expressions, Finite State Processes, and Three Problems of Equivalence, Inf. Comput., 86(1), 1990, 43-68.

[18] Kerstan, H., König, B.: Coalgebraic Trace Semantics for Continuous Probabilistic Transition Systems, Logical Methods in Computer Science, 9(4), 2013.

[19] Kock, A.: Monads and extensive quantities, 2011, ArXiv:1103.6009.

[20] Kock, A.: Commutative monads as a theory of distributions, Theory and Applications of Categories, 26(4), 2012, 97-131.

[21] Kwiatkowska, M., Parker, D.: Advances in Probabilistic Model Checking, Software Safety and Security Tools for Analysis and Verification (T. Nipkow, O. Grumberg, B. Hauptmann, Eds.), 33, IOS Press, 2012.

[22] Moss, L. S.: Coalgebraic Logic, Ann. Pure Appl. Logic, 96(1-3), 1999, 277-317.

[23] Pattinson, D.: Coalgebraic modal logic: soundness, completeness and decidability of local consequence, Theor. Comput. Sci., 309(1-3), 2003, 177-193.

[24] Rutten, J. J. M. M.: Universal coalgebra: a theory of systems, Theor. Comput. Sci., 249(1), 2000, 3-80.

[25] Sangiorgi, D.: On the origins of bisimulation and coinduction, ACM Trans. Program. Lang. Syst., 31(4), 2009. 